\title{
The Physicochemical and Antioxidant Properties of Sambucus nigra L. and Sambucus nigra Haschberg during Growth Phases: From Buds to Ripening
}

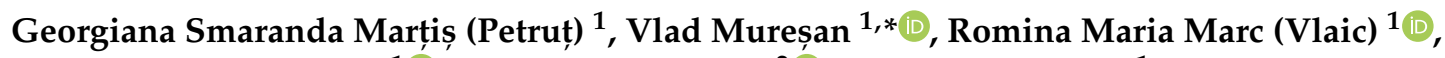

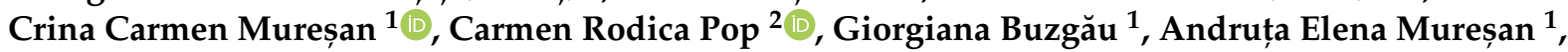 \\ Rodica Ana Ungur ${ }^{3, *}$ and Sevastița Muste ${ }^{1}$
}

1 Department of Food Engineering, Faculty of Food Science and Technology, University of Agricultural Sciences and Veterinary Medicine of Cluj-Napoca, 3-5 Mănăștur Street, 400372 Cluj-Napoca, Romania; georgiana.petrut@usamvcluj.ro (G.S.M.); romina.vlaic@usamvcluj.ro (R.M.M.); crina.muresan@usamvcluj.ro (C.C.M.); giorgiana_buzgau@yahoo.com (G.B.); andruta.muresan@usamvcluj.ro (A.E.M.); sevastita.muste@usamvcluj.ro (S.M.)

2 Department of Food Science, Faculty of Food Science and Technology, University of Agricultural Sciences and Veterinary Medicine of Cluj-Napoca, 3-5 Mănăștur Street, 400372 Cluj-Napoca, Romania; carmen-rodica.pop@usamvcluj.ro

3 Department of Rehabilitation Iuliu-Hațieganu, Faculty of General Medicine, University of Medicine and Pharmacy, 8 Victor Babeș Street, 400012 Cluj-Napoca, Romania

check for updates

Citation: Marțiș (Petruț), G.S.;

Mureșan, V.; Marc (Vlaic), R.M.; Mureșan, C.C.; Pop, C.R.; Buzgău, G.; Mureșan, A.E.; Ungur, R.A.; Muste, S. The Physicochemical and Antioxidant Properties of Sambucus nigra L. and Sambucus nigra Haschberg during Growth Phases: From Buds to Ripening. Antioxidants 2021, 10, 1093. https://doi.org/10.3390/ antiox10071093

Academic Editors: Filomena Nazzaro and Vincenzo De Feo

Received: 25 May 2021

Accepted: 2 July 2021

Published: 7 July 2021

Publisher's Note: MDPI stays neutral with regard to jurisdictional claims in published maps and institutional affiliations.

Copyright: () 2021 by the authors. Licensee MDPI, Basel, Switzerland. This article is an open access article distributed under the terms and conditions of the Creative Commons Attribution (CC BY) license (https:// creativecommons.org/licenses/by/ $4.0 /)$.
* Correspondence: vlad.muresan@usamvcluj.ro (V.M.); rodica.ana.ungur@gmail.com (R.A.U.); Tel.: +40-264-596384 (V.M.); +40-745-371502 (R.A.U.)

\begin{abstract}
Elderberry growth phases represent an irreversible process involving a series of biochemical changes that have an extremely important impact on nutritional characteristics. The aim was to assess the impact of genotype and maturity stage on phenolic compounds, antioxidant capacity and mineral profile in Sambucus plants harvested during different growth phases, from green elder flower buds to purple-black elderberries, including pollen, peduncles and seeds. Growth phases proved to have a greater influence compared to varieties. The green buds and flowers of both varieties had a high concentration of quercetin 3-rutinoside, also termed the key compound of the study. It was found that antioxidant activity varied in the following order: blooming elder flower pollen $>$ white elder flower buds > blooming elder flowers. Based on these findings, several novel food ingredients and supplements could be obtained in order to develop innovative health-promoting products.
\end{abstract}

Keywords: Sambucus nigra L.; elderberry ripening; antioxidant activity; polyphenols; health-promoting properties; rutin

\section{Introduction}

Sambucus nigra Haschberg (also known as standard cultivar of elderberry) and Sambucus nigra L. (also called European wild black elder) are some of the most versatile shrubs; native to the northern hemisphere, they belong to the Adoxaceae family in the order of the Dipsacales [1]. The comparison of major bioactive compounds and antioxidative properties of different selected types and wild plants of Sambucus nigra L. presents an interest in many areas, including horticulture [2], food industry [3], pharmaceutical industry [4] and, recently, in SARS-COV-2 antiviral drug research [5].

A new insight into elderberry anthocyanins bioactivity results from the exploring of anti-HCoV-NL63 activity of Sambucus Formosana Nakai stem ethanol extract and some markers of its phenolic compounds. It is has been demonstrated that Sambucus antiviral properties might be beneficial against the broad spectrum of human respiratory coronaviruses and applied for developing the antiviral agents in the future [6]. 
The chemical profile of the elderberry shrub is independently characterized whether one is talking about leaves, buds, berries, peduncles, flowers, pollen, roots, shoots, bark or seeds; although all these represent a complete life cycle of the Sambucus plant [7-10].

Fruit ripening affects several physicochemical parameters, resulting in changes in growth phases characteristics [11]. Research of the biochemical transformations underlying this process and their effects on several parts of Sambucus composition could provide valuable information on the stage of development of these plants suitable for formulating new food and cosmetic products.

The biochemistry of the elder fruit ripening is a genetic program that involves the degradation of chlorophyll, the biosynthesis of anthocyanins and their accumulation in vacuoles, increased cellular respiration mediated by mitochondrial enzymes and other biochemical changes [11].

Fruits of Sambucus commonly have a long ripening cycle. In general, one can divide the vegetation cycle in two periods, according to the most studied vegetative parts: the flower and then the fruits. Sambuci flores and Sambuci fructus present the most interest because they contain significant amounts of anthocyanins and other polyphenols [3,12,13].

The multitude of intermediate growth phases, with insufficient studies (green and red fruits, peduncles) [2] or no studies at all (elder buds), could improve the knowledge about bioactive compounds and minerals.

Sambucus peduncle waste resulting from the elderberry processing industry accounts for more than $10 \%$ of the total elderberry production and represents an extensive biochemical profile source of natural bioactive polyphenols with a potential low cost [1]. Actually, anthocyanins were first found in large amount in the peduncle, prior to berry ripening, and reached their highest concentrations after fruits had naturally detached. In fact, high amounts of anthocyanins compensate the xanthophyll cycle activity for decreasing the effects of light stress, throughout the Sambucus peduncles maturation process.

Each part of the vegetative Sambucus has a characteristic anthocyanin profile, mineral profile ( $\mathrm{Mg}, \mathrm{Ca}, \mathrm{Fe}, \mathrm{Zn}, \mathrm{Cu}$ and $\mathrm{Mn}$ ) [14] and bioactive compounds particularities, might be considered potential markers in food authenticity control. Additionally, to food implications, there is a growing interest in the use of new natural additives extracted from Sambucus fruits or flowers as a functional ingredient in food manufacturers, such as snacks enriched with extracts of Sambucus flowers, prepared by the extrusion process [15], inhibition of protein oxidation in meat and meat products with the addition of antioxidants from berries, or edible coatings [16].

Chemical composition of elder berry and flowers consists of primary metabolites (sugars, organic acids), secondary metabolites (phenols) and several other constituents (minerals, vitamins, cytokines). Secondary metabolites are susceptible to environmental and genetic growth factors and are well stated in metabolomic studies [11]. Thanks to these natural polyphenolic compounds, primarily flavonols, phenolic acids and anthocyanins, elder berry and flowers are characterized by high antioxidant activity [17]. These compounds are well known as free radical scavengers and are able to protect the human body against oxidative stress and lipid peroxidation (the literature search provided 7272 results for rutin-database PubMed).

Flavonoids were the most abundant polyphenols in elderberry fruit and were mainly represented by rutin $(813.08 \mu \mathrm{g} / 100 \mathrm{~g}$ dry weight). Rutin was the most important indicator of elderberry antioxidant activity $[1,12,18,19]$. Rutin content in vegetative organs of plants showed a good correlation with anthocyanins content [20]. A polyphenolic bioflavonoid, the rutin (chemically a glycoside comprising of flavonol aglycone quercetin along with disaccharide rutinose) has been reported for its various rheological effects [21]. Sambucus nigra extract, rich in rutin, extracted from wild Sambucus nigra elderflowers demonstrated the protective capacity for several degenerative diseases, such as inflammatory and cardiovascular disease, cancer, diabetes and nephrotoxicity induced by gentamicin [22].

Opposed to the chemical composition of elderberry fruit, which is especially rich in anthocyanins, flowers do not contain any pigments from this group. The concentration of 
flavonoids, however, is the highest in elderberry flowers [23]. According to the research of Dawidowicz et al. (2006) [23] elderflowers exhibit have a much stronger neutralizing activity of free radicals compared to elderberry fruit and DPPH values between $91.95 \%$ and $94.15 \%$ were are reported.

There is little information on the chemical composition during development among Sambucus species. To our knowledge, a characterization of the developmental phases from the flower bud to the ripe fruit was not made for the Sambucus nigra genotypes. Only few studies $[2,11]$ were focused on pre-harvest fruit or elderberry leaves. Therefore, the aim of this study was to assess the dynamics of total phenolic content and anthocyanins accumulation, and to assess the antioxidant activity during the growth and development of flowers and fruits from wild growing elderberry (Sambucus nigra L.) and cultivated elderberry (Sambucus nigra Haschberg L.) genotypes. Additionally, the level of several minerals ( $\mathrm{Ca}, \mathrm{Cd}, \mathrm{Cr}, \mathrm{Cu}, \mathrm{Fe}, \mathrm{K}, \mathrm{Mg}, \mathrm{Mn}, \mathrm{Na}, \mathrm{Ni}, \mathrm{Pb}$, Se and $\mathrm{Zn}$ ) was quantified during fruit development from fructification phase to ripe elderberry. In this regard, it is important to find out which of the vegetative parts of the wild/native elder could be relevant sources of secondary metabolites with high antioxidant activity possibly used in food formulations or in medicine.

\section{Materials and Methods}

\subsection{Experimental Field and Samples}

Two genotypes of Romanian organic Sambucus samples were collected in 2016: wild Sambucus nigra L. from Cluj-Napoca area, and cultivated Sambucus nigra Haschberg L. from an orchard village of Mânău, Maramureș. Figure 1 shows the aspects of the material studied, as well as the coding of the elder samples ( 36 samples) at different harvesting times, starting with the green elder flower buds phase (6 May 2016) until full maturity of elderflowers blooming (29 May 2016) and ending later with purple-black elderberries (5 September 2016) - with intermediate development phases. For each ripening phase, elder samples from two species were collected between 10 and 12 a.m. Moisture content was determined according to Wu et al. [24], while ash content was determined according to Kołodziej et al. [25]. For each type and sampling phases, buds/flowers/berries clusters were harvested whole, and the peduncles were removed manually later. The seeds were separated from frozen berries (green, red unripe elderberries seeds and purple-black elderberries seeds), as previously reported by Dulf et al. [10]. All samples were stored in polyethylene bags at $-18{ }^{\circ} \mathrm{C}$ until analysis. 

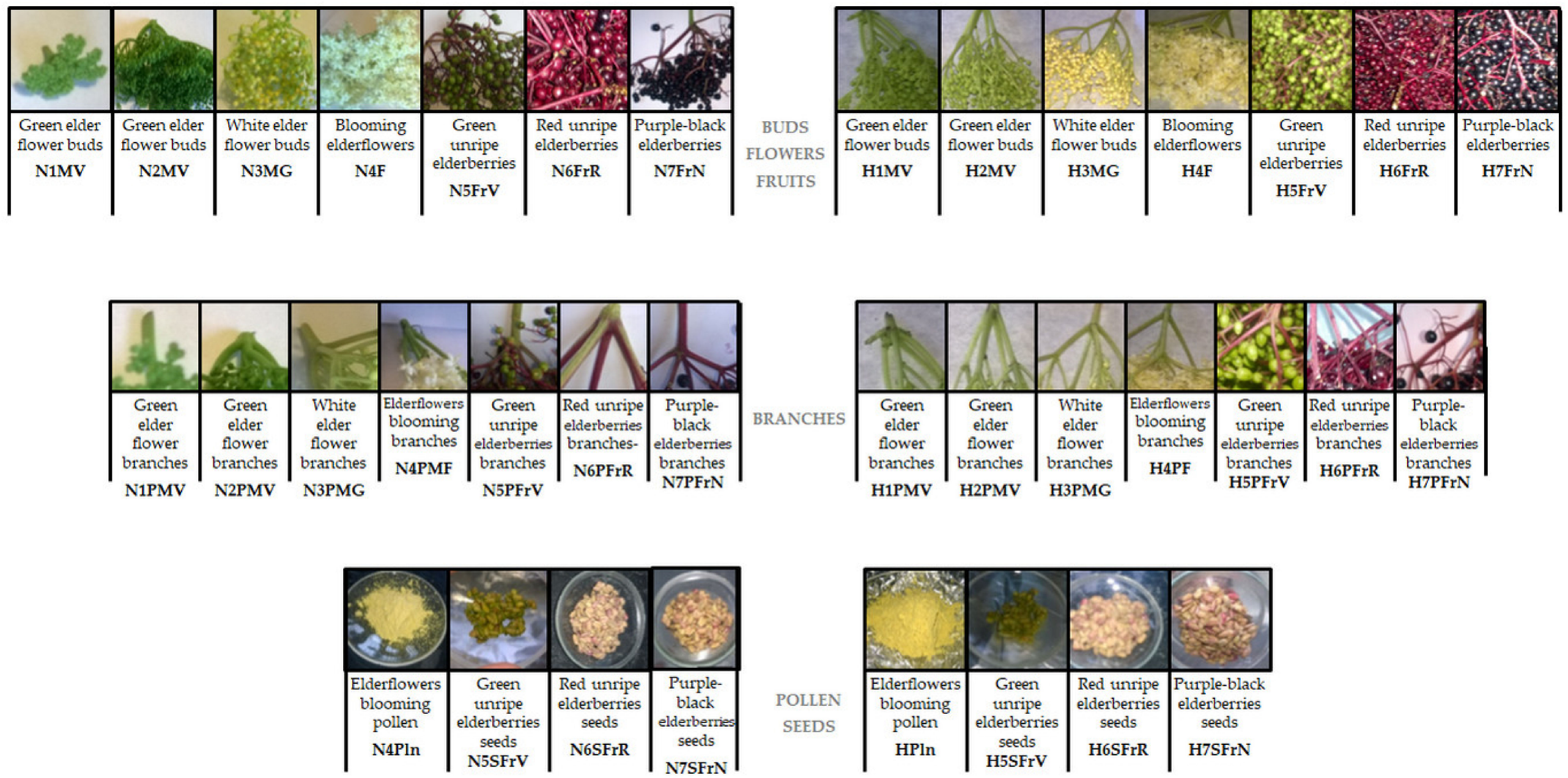

Wild growing genotypes

Cultivated genotypes

Figure 1. Sample coding. (H—“Haschberg” cultivars; N—wild “S. nigra L.”; M—buds; F—flowers; Fr—berries; P—peduncles; Pln—pollen; S—seeds). 


\subsection{Samples Extraction for Phytochemical Analysis}

The protocol for obtaining methanolic extracts from Transylvania elder samples followed the method proposed by Duymus et al. [26], with some minor modifications. The finely crushed sample of $1 \mathrm{~g}$ (frozen) was quickly homogenized with $100 \mathrm{~mL}$ of methanol and stirred using a Sigma model centrifuge model 2-5 (4000 rpm for $10 \mathrm{~min}$ ). The extraction operation was continued by soaking in the dark, for $24 \mathrm{~h}$, at a temperature of $3-6{ }^{\circ} \mathrm{C}$. The resulting supernatant was concentrated at $35^{\circ} \mathrm{C}$ under reduced pressure (Rotavap Laborata 4010 Digital, Heidolph). The extract obtained by drying was recovered in $10 \mathrm{~mL}$ of $\mathrm{MeOH}$ and stored at $-18{ }^{\circ} \mathrm{C}$. Prior to storage, the resulting extract was filtered using $0.45 \mu \mathrm{m}$ Millipore filters.

\subsection{Determination of Total Phenolic Content (TPC) by Folin-Ciocâlteu Method}

The quantification of polyphenols was performed according to the Folin-Ciocâlteu spectrophotometric method, described by Mikulic-Petkovsek et al. [19]. For this, over $25 \mu \mathrm{L}$ of methanolic extract, $1.8 \mathrm{~mL}$ of distilled water and $120 \mu \mathrm{L}$ of Folin-Ciocâlteu reagent were added; in order to create a $\mathrm{pH} \sim 10$, at an interval of $5 \mathrm{~min}, 340 \mu \mathrm{L}$ of $\mathrm{Na}_{2} \mathrm{CO}_{3}$ solution $(7.5 \%)$ was added to achieve the reaction between the phenolic compounds and the reagent used. After incubation of the samples for $90 \mathrm{~min}$ at room temperature, the absorbance was read using a Shimadzu UV-VIS 1700 spectrophotometer at $750 \mathrm{~nm}$. The reference sample was methanol. The determination was performed in triplicate.

\subsection{Determination and Quantification of Total Flavonoid Content (HPLC Analysis)}

The concentration of total flavonoids in ripening elder samples was performed by the colorimetric method described by Diaconeasa et al. [27]. The first step was to dilute the methanolic extracts with distilled water to a volume of $5 \mathrm{~mL}$ and add $300 \mu \mathrm{L}$ of $\mathrm{NaNO}_{2}$ $(5 \%)$. After $5 \mathrm{~min}$, the mixture was homogenized with $300 \mu \mathrm{L} \mathrm{AlCl} 3(10 \%)$ and after another 6 min with $2 \mathrm{~mL} \mathrm{NaOH}(1 \mathrm{~N})$. Then, the absorbance was read, using a UV-VIS 1700 Shimadzu spectrophotometer at $500 \mathrm{~nm}$. The quantification of flavonoids content was performed using the HPLC-ESI-MS method, described by Bunea et al. [28], with some modifications. The mobile phase represented by the mixture of water and $0.1 \%$ acetic acid, meanwhile the mobile phase $\mathrm{B}$ consisting of acetonitrile and the same amount of acetic acid. Both of them have been subjected, on the Eclipse XDB C18 column, with gradient parameters (B from $5 \%$ to $40 \%$ (0-18 $\mathrm{min}$ ), B from $40 \%$ to $90 \%$ (8-24 min), B from $90 \%$ to $5 \%$ (24-30 min), flow rate of $0.5 \mathrm{~mL} /$ minute, at $25{ }^{\circ} \mathrm{C}$, in order to distinguish phenolic compounds from elder. The wavelengths of the chromatograms were 280, 340 and $520 \mathrm{~nm}$, and the spectrum included the same values for peaks, in the range of $200-600 \mathrm{~nm}$. Positive ionization with working conditions: nitrogen flow $8 \mathrm{~L} /$ minute, capillary voltage $3000 \mathrm{~V}$, $\mathrm{m} / z$ 100-1000 full-scan, working temperature $300{ }^{\circ} \mathrm{C}$. The data interpretation software was represented by Agilent ChemStation.

\subsection{Determination of DPPH Free Radical Scavenging Capacity}

The method proposed by Diaconeasa et al. [27], with some modifications, allowed the estimation of the free radical reduction capacity using a solution of 2,2-diphenyl-1picrilhydrazyl (DPPH) and was adapted to evaluate the antioxidant activity of the different ripening elder extracts. A total of $100 \mu \mathrm{L}$ of the methanolic extract of the samples was added to the $3.9 \mathrm{~mL}$ DPPH solution $(0.025 \mathrm{~g} / \mathrm{L})$ to be analyzed. The mixture was properly homogenized, and kept in the dark for $30 \mathrm{~min}$. The absorbance of the samples was recorded at $515 \mathrm{~nm}$ (UV-VIS 1700 Shimadzu). The control solution was prepared according to the same protocol but replaced the $100 \mu \mathrm{L}$ of sample (methanolic extract) with methanol. The results were evaluated as a percentage of the inhibition capacity, based on the following equation: DPPH scavenging effect $(\%)=[(\mathrm{Ac}-\mathrm{AP}) \times 100] / \mathrm{Ac}]$, where: Ac-absorbance of the control solution (nm); $\mathrm{AP}$-sample absorbance $(\mathrm{nm})$. 


\subsection{Mineral Estimation by Atomic Absorption Spectrometry Method (AAS)}

The evaluation of micro- and macro-elements $(\mathrm{Ca}, \mathrm{Cd}, \mathrm{Cr}, \mathrm{Cu}, \mathrm{Fe}, \mathrm{K}, \mathrm{Mg}, \mathrm{Mn}, \mathrm{Na}$, $\mathrm{Ni}, \mathrm{Pb}$, Se and $\mathrm{Zn}$ ) by atomic absorption spectrometry (AAS), was performed according to the method described by Pasca et al. [29]. The equipment used was the Analyst 800 spectrometer heaving a graphite furnace transverse by electric heating. The applied voltage is transverse to a tube, adjacent to the light beam, and towards the end, the electromagnet creates a magnetic field whose trajectory is parallel to the beam propagated from the lamp. The analysis sample went through three stages (drying, followed by pyrolysis and atomization), due to the rising temperature of the tube.

The mineralization of the plant matrices was performed using the calcination furnace Berghof microwave digestion system MWS-2. Amounts of $0.3 \pm 0.01 \mathrm{~g}$ of the crushed sample were mixed with $2 \mathrm{~mL}$ of $\mathrm{HNO}_{3}$ solution $(65 \%)$, followed by 15 min rest time. Before sealing the sample containers, $3 \mathrm{~mL}$ of $\mathrm{H}_{2} \mathrm{O}_{2}$ were added.

At the end of the initial program the solution was transferred at the end of the initial program in graduated containers. In the case of elder samples, the content was brought up to a gradation of $125 \mathrm{~mL}$ with ultrapure water. Injection of elder samples into SAA with AS-800 auto sampler was performed immediately after mineralization. Regarding the tracing of the calibration curve, calcium $(2 \mu \mathrm{g} / \mathrm{L})$, cadmium $(2.0 \mu \mathrm{g} / \mathrm{L})$, chromium $(10 \mu \mathrm{g} / \mathrm{L})$, copper $(25 \mu \mathrm{g} / \mathrm{L})$, iron $(20 \mu \mathrm{g} / \mathrm{L})$, potassium $(5.0 \mu \mathrm{g} / \mathrm{L})$, magnesium $(1.0 \mu \mathrm{g} / \mathrm{L})$, manganese $(10 \mu \mathrm{g} / \mathrm{L})$, sodium $(4.0 \mu \mathrm{g} / \mathrm{L})$, nickel $(50 \mu \mathrm{g} / \mathrm{L})$, lead $(50 \mu \mathrm{g} / \mathrm{L})$, selenium $(100 \mu \mathrm{g} / \mathrm{L})$ and zinc $(2.0 \mu \mathrm{g} / \mathrm{L})$ were used as dilutions of standard solutions. The results were expressed as arithmetic mean value, in $\mathrm{mg} / \mathrm{kg}$ sample.

\subsection{Statistical Analysis}

The main component analysis (PCA) was performed by The Unscrambler X 10.5.1 software. Data were reported as average mean \pm standard deviation (SD) for triplicate determinations. The ANOVA analysis of variance was used to compare the average mean values, using SPSS 19.0 statistical analysis (IBM, New York, USA) and Tukey's Honestly Significant Differences (HSD) test with a confidence interval of $95 \%$ or $99 \%$. A $p$-value below 0.05 was considered statistically significant. The intensity of relationships between the DPPH and acid chlorogenic, flavonols values, between total phenols and flavonoids, were determined with Pearson's correlation with a 95\% confidence interval.

\section{Results}

\subsection{Total Phenolic Content (TPC)}

The differences in TPC between elderberry genotypes and the fructification stages are indicated in Table 1. The trend of the developmental stages of elderberry fruit regarding TPC varied between the samples as follows: in the early developmental phase of the bud, there was a significant value of polyphenols for both genotypes (N1MV $565.445 \pm 0.003 \mathrm{mg}$ GAE/100 g, H1MV $570.442 \pm 0.00 \mathrm{mg}$ GAE/100 g), which increased during the evolution of the bud until before the onset of blooming $(883.066 \pm 0.001 \mathrm{mg} \mathrm{GAE} / 100 \mathrm{~g}$ N3MG, $890.04 \pm 0.00 \mathrm{mg} \mathrm{GAE} / 100 \mathrm{~g} \mathrm{H} 3 \mathrm{MG})$. The elder flower blooming stage (N4F and H4F) announced a decline in TPC content; the decrease of TPC $(p<0.001)$ continued during the green unripe elderberry ( $\mathrm{N} 5 \mathrm{FrV}$ and $\mathrm{H} 5 \mathrm{FrV})$ and red unripe elderberry stage (N6FrR and H6FrR). 
Table 1. Antioxidant activity, total flavonoids and phenols of different elderberry genotypes during developmental phases.

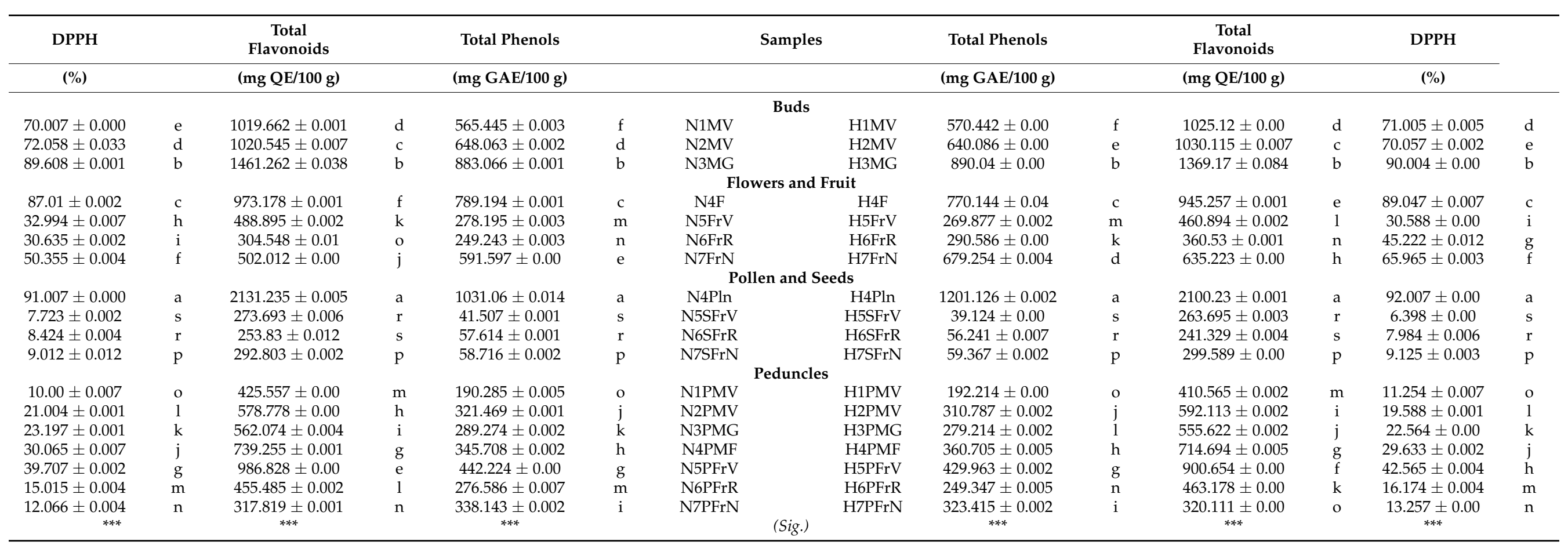

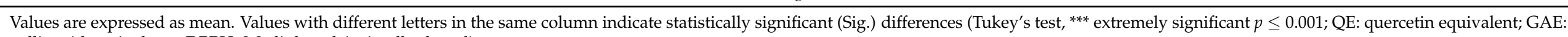
gallic acid equivalents; DPPH: 2,2-diphenyl-1-picrylhydrazyl) 


\subsection{Total Flavonoid Content (TFC) in Different Growth Phases of Elderberry Fruit}

The profile of flavonoids in the seven developmental phases of elderberry buds, flowers and fruit (Table 1) generally indicates higher total flavonoid values in elder flower buds, while in the fruit formation stages, it involves distinct variations. Flavonoid accumulation follows the same rhythm for both studied varieties. It is important to mention the fact that flavonoids significantly contribute to a good adaptation of plants to unpredictable environmental conditions. Flavonoid biosynthetic pathways are strongly inducible, and are especially sensitive to biotic stimuli, including elicitation by bacteria [30]. White elder flower buds (N3MG and H3MG), pollen (N4Pln and H4Pln) and green elderberry peduncles (N5PFrV and H5PFrV) are of interest due to their flavonoid compound content. This study indicates the potential of bioactive compounds in the buds and peduncles of the elderberry, as unexploited plant parts.

\subsection{Antioxidant Activity in Different Phases of Elderberry Development}

The various part extracts from the wild or cultivated elderberry displayed strong antioxidant activities, especially for pollen (N4Pln and H4Pln), followed by white elder flower buds (N3MG and H3MG) and blooming elder flowers (N4F and H4F). Green elderberry seeds (N5SFrV and $\mathrm{H} 5 \mathrm{SFrV}$ ) show the weakest antioxidant action in the analyzed samples $(p \leq 0.001)$ (Table 1$)$.

\subsection{Phenolic Compound Profile by HPLC-ESI-MS}

The concentrations of individual anthocyanins, flavonols and hydroxycinnamic acids are reported in Table 2. A total of 13 compounds were identified in the wild/native grown samples by the HPLC-ESI-MS method (similarity of the retention time and UV spectra to commercial standards), of which 11 compounds were detected in all developing phenophases: (1) cyanidin 3-sambubioside-5-glucoside, (3) cyanidin 3,5-diglucoside, (4) procyanidin dimer isomer 1, (6) chlorogenic acid, (7) catechin, (8) epicatechin, (9) procyanidin dimer isomer 2, (10) quercetin 3-rutinoside, (11) quercetin 3-glucoside and (12) kaempferol-3-rutinoside.

The most frequent anthocyanin group in the studied elderberry varieties are cyanidin glycosides (MS2 fragmentation at $m / z, 743,581,449,287$, Table 2, Figure 2). The representatives of anthocyanins detected in both varieties included cyanidin 3-sambubioside-5glucoside, cyanidin 3-sambubioside, cyanidin 3,5-diglucoside and cyanidin 3-glucoside. The content of these compounds during fructification has an ascending evolution, except for cyanidin 3-sambubioside. Across the developmental stages of the elderberry, in the purple-black elderberry phase of both varieties (N7FrN si H7FrN), maximum values of anthocyanic compounds occur due to accumulation of phenylpropanoid enzymes. 
Table 2. Representative flavonoids in methanolic extracts from Sambucus nigra L. and Sambucus nigra Haschberg, in different growth phases of the fruit.

\begin{tabular}{|c|c|c|c|c|c|c|c|c|c|c|c|c|c|c|c|c|}
\hline Nr. Peak & 1 & 2 & 3 & 4 & 5 & 6 & 7 & 8 & 9 & 10 & 11 & 12 & 13 & \multirow{4}{*}{\multicolumn{3}{|c|}{ Amount of $(\mu \mathrm{g} / \mathrm{mL})$}} \\
\hline $\begin{array}{l}\text { retention } \\
\text { Time } \\
\text { (min.) }\end{array}$ & 3.01 & 3.47 & 4.02 & 10.27 & 11.55 & 12.09 & 12.37 & 13.38 & 13.74 & 15.67 & 16.40 & 16.81 & 17.67 & & & \\
\hline$\frac{[\mathrm{M}-\mathrm{H}]+}{(\mathrm{m} / \mathrm{z})}$ & $\begin{array}{l}743 ; 581 ; \\
449 ; 287\end{array}$ & $581 ; 287$ & $\begin{array}{l}\text { 611; } 449 ; \\
287\end{array}$ & 579 & $449 ; 287$ & $355 ; 181$ & 291 & 291 & 579 & $611 ; 303$ & $465 ; 303$ & $595 ; 287$ & 579 & & & \\
\hline $\begin{array}{l}\mathrm{UV} \lambda_{\max } \\
(\mathrm{nm})\end{array}$ & $520 ; 280$ & $520 ; 280$ & $\begin{array}{l}520 ; 350 ; \\
280\end{array}$ & 279 & $520 ; 280$ & $330 ; 280$ & 280 & 280 & 279 & $360 ; 270$ & $360 ; 270$ & $356 ; 264$ & 279 & & & \\
\hline \multirow[t]{2}{*}{ 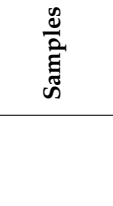 } & $\begin{array}{l}\text { Cy 3-sam- } \\
\text { 5-gluc }\end{array}$ & Cy 3-sam & $\begin{array}{c}\text { Cy } \\
\text { 3,5-digluc }\end{array}$ & $\begin{array}{l}\text { Procyan- } \\
\text { DI }\end{array}$ & Cyan-G & $\begin{array}{c}\text { Chlorog } \\
\text { ac }\end{array}$ & Catech & Epicatech & $\begin{array}{c}\text { Procyan-D } \\
\text { II }\end{array}$ & $\begin{array}{l}\text { Quercet- } \\
\text { Ru }\end{array}$ & Q 3-gluc & K 3-rut & $\begin{array}{c}\text { Procyan-D } \\
\text { III }\end{array}$ & Anthocy & Flav & $\underset{\text { ac }}{\text { Hydroxyc }}$ \\
\hline & & & & & & & & & & & & & & $\begin{array}{c}1+2+3+ \\
5\end{array}$ & $\begin{array}{c}4+7+8+ \\
9+10+11 \\
+12+13\end{array}$ & 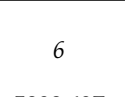 \\
\hline N1MV & $\begin{array}{c}40.783 \pm \\
0.002 \mathrm{~g}\end{array}$ & $\begin{array}{c}33.788 \pm \\
0.002 \mathrm{~g}\end{array}$ & $\begin{array}{c}28.891 \pm \\
0.002 \mathrm{i}\end{array}$ & $\begin{array}{c}244.953 \pm \\
0.002 \mathrm{~g}\end{array}$ & $\mathrm{Nd}$ & $\begin{array}{l}5808.697 \\
\pm 0.002 \mathrm{c}\end{array}$ & $\begin{array}{c}125.758 \pm \\
0.002 \mathrm{j}\end{array}$ & $\begin{array}{c}112.941 \pm \\
0.002 \mathrm{j}\end{array}$ & $\begin{array}{c}116.145 \pm \\
0.002 \mathrm{j}\end{array}$ & $\begin{array}{c}905.806 \pm \\
0.002 \mathrm{~h}\end{array}$ & $\begin{array}{r}73.45 \pm \\
0.002 \mathrm{e}\end{array}$ & $\begin{array}{c}182.837 \\
\pm 0.002 \mathrm{~d}\end{array}$ & $\begin{array}{c}317.368 \pm \\
0.002 \mathrm{c}\end{array}$ & $\begin{array}{c}103.462 \pm \\
0.006\end{array}$ & $\begin{array}{c}2079.231 \\
\pm 0.016\end{array}$ & $\begin{array}{c}5808.697 \\
\pm 0.002\end{array}$ \\
\hline H1MV & $\begin{array}{c}40.545 \pm \\
0.002 \mathrm{~h}\end{array}$ & $\mathrm{Nd}$ & $\begin{array}{r}29.53 \pm \\
0.002 \mathrm{i}\end{array}$ & $\begin{array}{c}181.152 \pm \\
0.002 \mathrm{j}\end{array}$ & $\mathrm{Nd}$ & $\begin{array}{l}10257.656 \\
\pm 0.002 \mathrm{a}\end{array}$ & $\begin{array}{c}129.718 \pm \\
0.002 \mathrm{~h}\end{array}$ & $\begin{array}{c}140.135 \pm \\
0.002 \mathrm{~g}\end{array}$ & $\begin{array}{c}142.739 \pm \\
0.002 \mathrm{e}\end{array}$ & $\begin{array}{c}4057.00 \pm \\
0.002 \mathrm{~b}\end{array}$ & $\begin{array}{c}73.15 \pm \\
0.002 \mathrm{f}\end{array}$ & $\begin{array}{c}410.596 \\
\pm 0.002 \mathrm{c}\end{array}$ & $\begin{array}{c}310.062 \pm \\
0.002 \mathrm{~d}\end{array}$ & $\begin{array}{c}70.075 \pm \\
0.004\end{array}$ & $\begin{array}{c}5444.552 \\
\pm 0.016\end{array}$ & $\begin{array}{c}10,257.656 \\
\pm 0.002\end{array}$ \\
\hline $\mathrm{N} 4 \mathrm{~F}$ & $\begin{array}{c}33.508 \pm \\
0.002 j\end{array}$ & $\mathrm{Nd}$ & $\begin{array}{r}31.728 \pm \\
0.002 \mathrm{~g}\end{array}$ & $\begin{array}{c}259.168 \pm \\
0.002 \mathrm{f}\end{array}$ & $\begin{array}{c}77.839 \pm \\
0.002 \mathrm{~h}\end{array}$ & $\begin{array}{l}4948.771 \\
\pm 0.002 \mathrm{~d}\end{array}$ & $\begin{array}{c}160.652 \pm \\
0.002 \mathrm{~g}\end{array}$ & $\begin{array}{c}162.609 \pm \\
0.002 \mathrm{e}\end{array}$ & $\begin{array}{c}139.775 \pm \\
0.002 \mathrm{~g}\end{array}$ & $\begin{array}{l}1983.462 \\
\pm 0.002 \mathrm{c}\end{array}$ & $\begin{array}{c}127.141 \\
\pm 0.002 \mathrm{~b}\end{array}$ & $\begin{array}{c}520.236 \\
\pm 0.002 \mathrm{~b}\end{array}$ & $\begin{array}{c}1827.588 \pm \\
0.002 \mathrm{~b}\end{array}$ & $\begin{array}{c}143.075 \pm \\
0.006\end{array}$ & $\begin{array}{c}5180.588 \\
\pm 0.016\end{array}$ & $\begin{array}{c}4948.771 \\
\pm 0.002\end{array}$ \\
\hline $\mathrm{H} 4 \mathrm{~F}$ & $\begin{array}{c}37.916 \pm \\
0.002 \mathrm{i}\end{array}$ & $\mathrm{Nd}$ & $\begin{array}{c}29.787^{\circ} \pm \\
0.002 \mathrm{~h}\end{array}$ & $\begin{array}{c}358.277 \pm \\
0.002 \mathrm{c}\end{array}$ & $\begin{array}{c}121.287 \pm \\
0.002 \mathrm{~g}\end{array}$ & $\begin{array}{l}7429.495 \\
\pm 0.002 \mathrm{~b}\end{array}$ & $\begin{array}{c}195.378 \pm \\
0.002 \mathrm{~d}\end{array}$ & $\begin{array}{c}202.984 \pm \\
0.002 \mathrm{~b}\end{array}$ & $\begin{array}{c}147.205 \pm \\
0.002 \mathrm{~d}\end{array}$ & $\begin{array}{l}6178.463 \\
\pm 0.002 \mathrm{a}\end{array}$ & $\begin{array}{c}209.863 \\
\pm 0.002 \mathrm{a}\end{array}$ & $\begin{array}{l}1244.874 \\
\pm 0.002 \mathrm{a}\end{array}$ & $\begin{array}{c}2913.322 \pm \\
0.002 \mathrm{a}\end{array}$ & $\begin{array}{c}188.99 \pm \\
0.006\end{array}$ & $\begin{array}{l}11,450.366 \\
\quad \pm 0.016\end{array}$ & $\begin{array}{c}7429.495 \\
\pm 0.002\end{array}$ \\
\hline N5FrV & $\begin{array}{r}53.048 \pm \\
0.002 \mathrm{f}\end{array}$ & $\begin{array}{r}34.972 \pm \\
0.002 \mathrm{f}\end{array}$ & $\begin{array}{r}31.843 \pm \\
0.002 \mathrm{f}\end{array}$ & $\begin{array}{c}206.516 \pm \\
0.002 \mathrm{i}\end{array}$ & $\begin{array}{c}228.418 \pm \\
0.002 \mathrm{e}\end{array}$ & $\begin{array}{l}2072.697 \\
\pm 0.002 \mathrm{e}\end{array}$ & $\begin{array}{c}173.396 \pm \\
0.002 \mathrm{f}\end{array}$ & $\begin{array}{c}191.229 \pm \\
0.002 \mathrm{~d}\end{array}$ & $\begin{array}{c}198.236 \pm \\
0.002 \mathrm{c}\end{array}$ & $\begin{array}{l}1254.869 \\
\pm 0.002 \mathrm{f}\end{array}$ & $\begin{array}{r}51.402 \pm \\
0.002 \mathrm{i}\end{array}$ & $\begin{array}{r}57.882 \pm \\
0.002 \mathrm{~g}\end{array}$ & $\begin{array}{r}95.691 \pm \\
0.002 \mathrm{~g}\end{array}$ & $\begin{array}{c}348.281 \pm \\
0.008\end{array}$ & $\begin{array}{c}2229.221 \\
\pm 0.016\end{array}$ & $\begin{array}{c}2072.697 \\
\pm 0.002\end{array}$ \\
\hline N6FrR & $\begin{array}{r}92.338 \pm \\
0.002 \mathrm{c}\end{array}$ & $\begin{array}{l}40.903 \pm \\
0.002 \mathrm{~d}\end{array}$ & $\begin{array}{r}76.132 \pm \\
0.002 \mathrm{c}\end{array}$ & $\begin{array}{c}350.014 \pm \\
0.002 \mathrm{~d}\end{array}$ & $\begin{array}{c}277.996 \pm \\
0.002 \mathrm{~d}\end{array}$ & $\begin{array}{c}302.247 \pm \\
0.002 \mathrm{i}\end{array}$ & $\begin{array}{c}191.222 \pm \\
0.002 \mathrm{e}\end{array}$ & $\begin{array}{c}140.873 \pm \\
0.002 \mathrm{f}\end{array}$ & $\begin{array}{c}142.164 \pm \\
0.002 \mathrm{f}\end{array}$ & $\begin{array}{c}256.771 \pm \\
0.002 \mathrm{i}\end{array}$ & $\begin{array}{c}73.984 \pm \\
0.002 \mathrm{~d}\end{array}$ & $\begin{array}{c}72.89 \pm \\
0.002 \mathrm{e}\end{array}$ & $\begin{array}{c}98.916 \pm \\
0.002 \mathrm{f}\end{array}$ & $\begin{array}{c}487.369 \pm \\
0.008\end{array}$ & $\begin{array}{c}1326.834 \\
\pm 0.016\end{array}$ & $\begin{array}{l}302.247 \\
\pm 0.002\end{array}$ \\
\hline H6FrR & $\begin{array}{r}74.825 \pm \\
0.002 \mathrm{~d}\end{array}$ & $\begin{array}{r}41.469 \pm \\
0.002 \mathrm{c}\end{array}$ & $\begin{array}{r}37.607 \pm \\
0.002 \mathrm{~d}\end{array}$ & $\begin{array}{c}259.423 \pm \\
0.002 \mathrm{e}\end{array}$ & $\begin{array}{c}680.508 \pm \\
0.002 \mathrm{c}\end{array}$ & $\begin{array}{c}884.757 \pm \\
0.002 \mathrm{~h}\end{array}$ & $\begin{array}{c}384.233 \pm \\
0.002 \mathrm{c}\end{array}$ & $\begin{array}{c}200.235 \pm \\
0.002 \mathrm{c}\end{array}$ & $\begin{array}{c}216.962 \pm \\
0.002 \mathrm{~b}\end{array}$ & $\begin{array}{l}1407.889 \\
\pm 0.002 \mathrm{e}\end{array}$ & $\begin{array}{r}65.738 \pm \\
0.002 \mathrm{~g}\end{array}$ & $\begin{array}{c}49.011 \pm \\
0.002 j\end{array}$ & $\mathrm{Nd}$ & $\begin{array}{c}834.409 \pm \\
0.008\end{array}$ & $\begin{array}{c}2583.491 \\
\pm 0.014\end{array}$ & $\begin{array}{l}884.757 \\
\pm 0.002\end{array}$ \\
\hline N7FrN & $\begin{array}{c}260.971 \pm \\
0.002 \mathrm{~b}\end{array}$ & $\begin{array}{c}82.463 \pm \\
0.002 \mathrm{~b}\end{array}$ & $\begin{array}{c}156.729 \pm \\
0.002 \mathrm{~b}\end{array}$ & $\begin{array}{l}1361.398 \\
\pm 0.002 \mathrm{~b}\end{array}$ & $\begin{array}{l}3045.063 \\
\pm 0.002 \mathrm{~b}\end{array}$ & $\begin{array}{c}99.587 \pm \\
0.002 \mathrm{j}\end{array}$ & $\begin{array}{c}129.298 \pm \\
0.002 \mathrm{i}\end{array}$ & $\begin{array}{c}131.723 \pm \\
0.002 \mathrm{~h}\end{array}$ & $\begin{array}{c}127.479 \pm \\
0.002 \mathrm{~h}\end{array}$ & $\begin{array}{c}158.603 \pm \\
0.002 j\end{array}$ & $\begin{array}{c}61.958 \pm \\
0.002 \mathrm{~h}\end{array}$ & $\begin{array}{r}59.901 \pm \\
0.002 \mathrm{f}\end{array}$ & $\mathrm{Nd}$ & $\begin{array}{c}3545.226 \\
\pm 0.008\end{array}$ & $\begin{array}{r}2030.303 \\
\pm 0.014\end{array}$ & $\begin{array}{l}99.587 \\
\pm 0.002\end{array}$ \\
\hline H7FrN & $\begin{array}{c}332.164 \pm \\
0.002 \mathrm{a}\end{array}$ & $\begin{array}{c}121.412 \pm \\
0.002 \mathrm{a}\end{array}$ & $\begin{array}{c}170.991 \pm \\
0.002 \mathrm{a}\end{array}$ & $\begin{array}{l}1479.139 \\
\pm 0.002 \mathrm{a}\end{array}$ & $\begin{array}{l}4611.039 \\
\pm 0.002 \mathrm{a}\end{array}$ & $\begin{array}{c}923.757 \pm \\
0.002 \mathrm{~g}\end{array}$ & $\begin{array}{c}386.604 \pm \\
0.002 \mathrm{~b}\end{array}$ & $\begin{array}{c}118.635 \pm \\
0.002 \mathrm{i}\end{array}$ & $\begin{array}{c}122.281 \pm \\
0.002 \mathrm{i}\end{array}$ & $\begin{array}{c}948.865 \pm \\
0.002 \mathrm{~g}\end{array}$ & $\begin{array}{c}118.758 \\
\pm 0.002 \mathrm{c}\end{array}$ & $\begin{array}{c}51.443 \pm \\
0.002 \mathrm{i}\end{array}$ & $\begin{array}{l}\mathrm{Nd} \\
* * *\end{array}$ & $\begin{array}{c}5235.606 \\
\pm 0.002\end{array}$ & $\begin{array}{c}3225.725 \\
\pm 0.014\end{array}$ & $\begin{array}{l}923.757 \\
\pm 0.002\end{array}$ \\
\hline (Sig.) & $* * *$ & $* * *$ & $* * *$ & $* * *$ & $* * *$ & $* * *$ & $* * *$ & $* * *$ & $* * *$ & $* * *$ & $* * *$ & $* * *$ & $* * *$ & & & \\
\hline
\end{tabular}

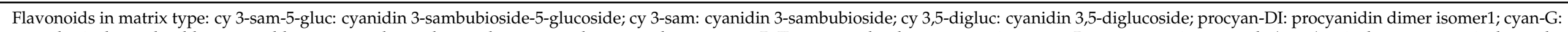

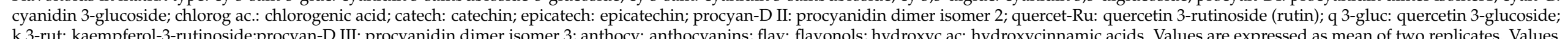

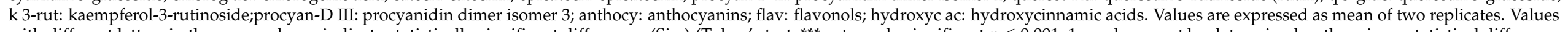

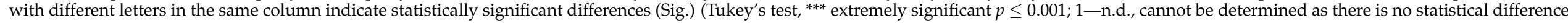
between the results. 


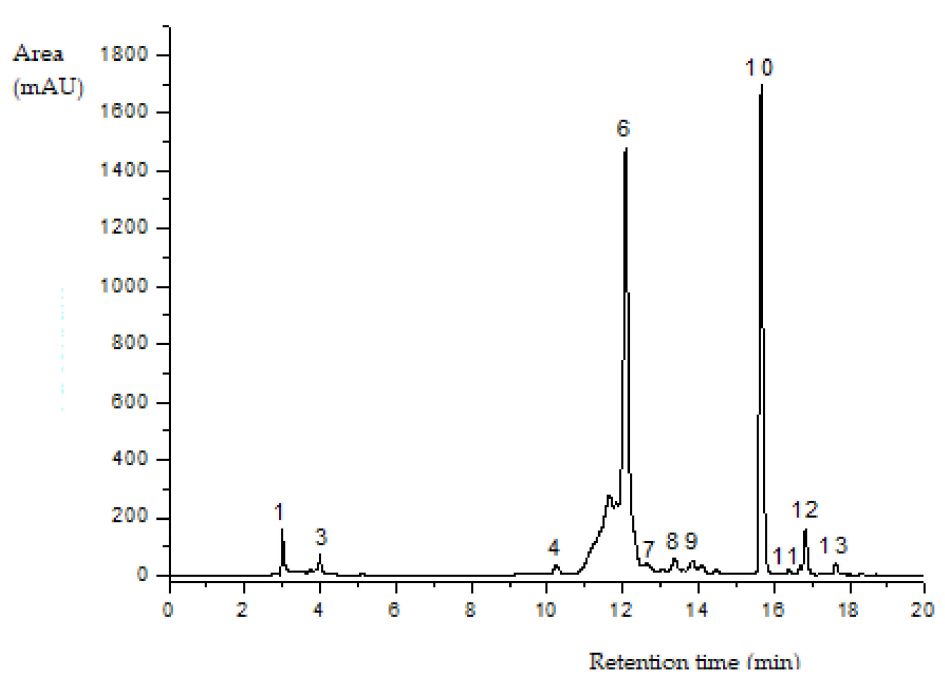

(a1). H1MV $280 \mathrm{~nm}$

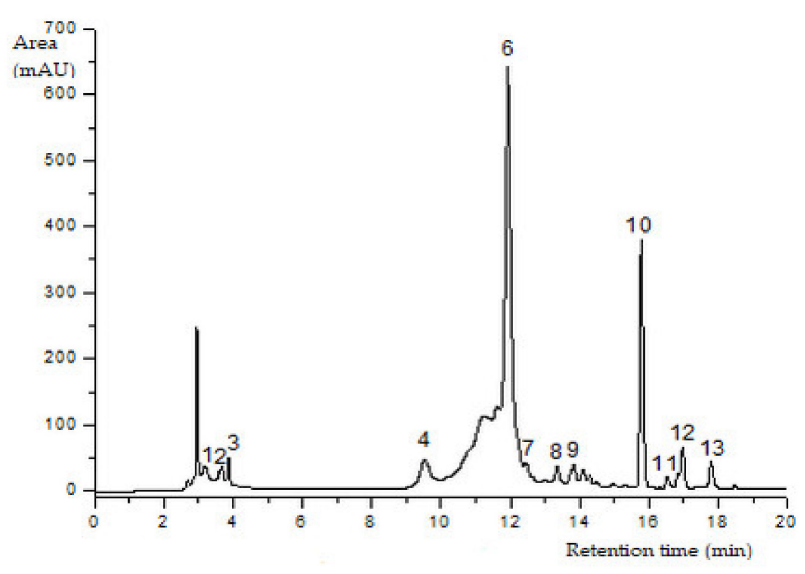

(a2). N1MV $280 \mathrm{~nm}$

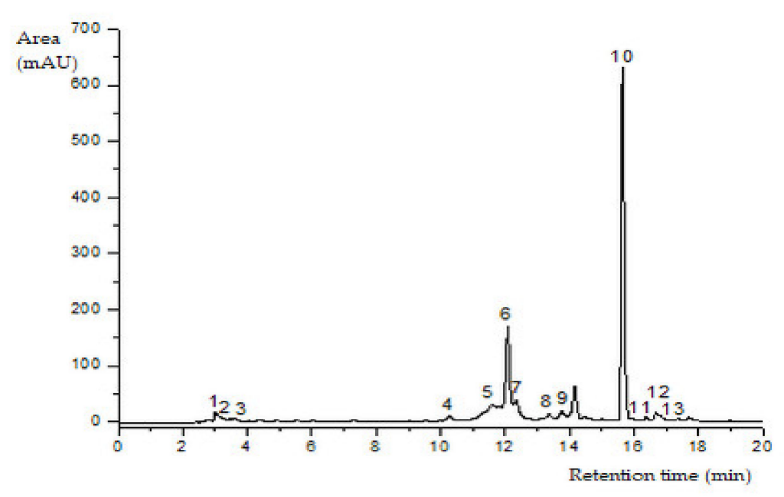

(c1). H5FrV280 nm

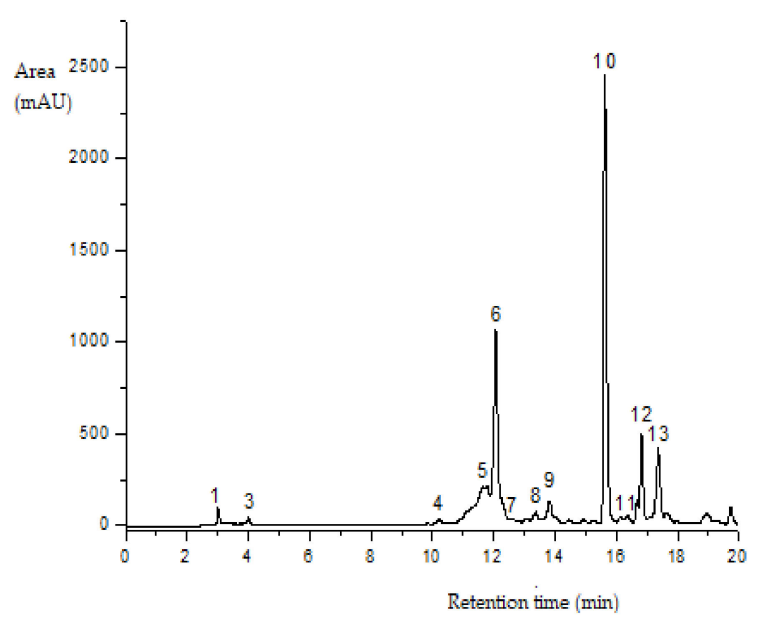

(b1). H4F $280 \mathrm{~nm}$

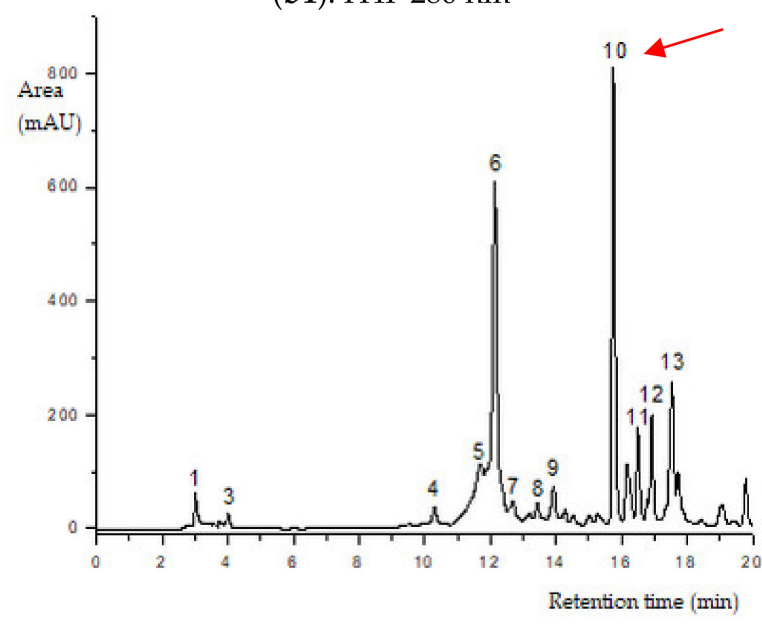

(b2). N4F $280 \mathrm{~nm}$

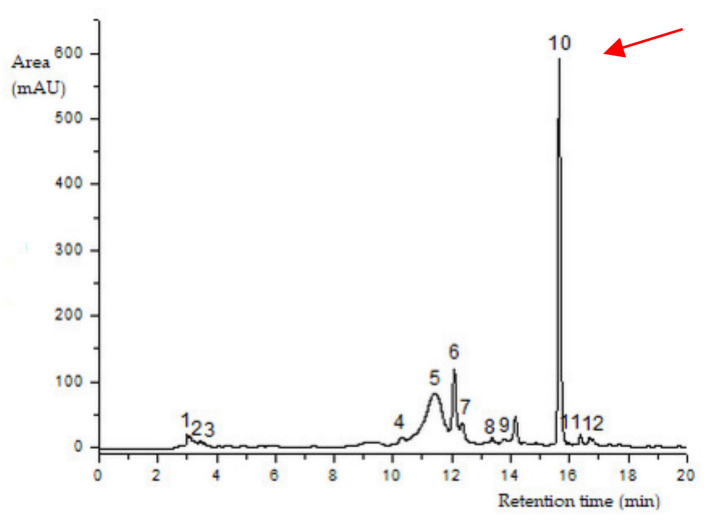

(d1). H6FrR $280 \mathrm{~nm}$

Figure 2. Cont. 


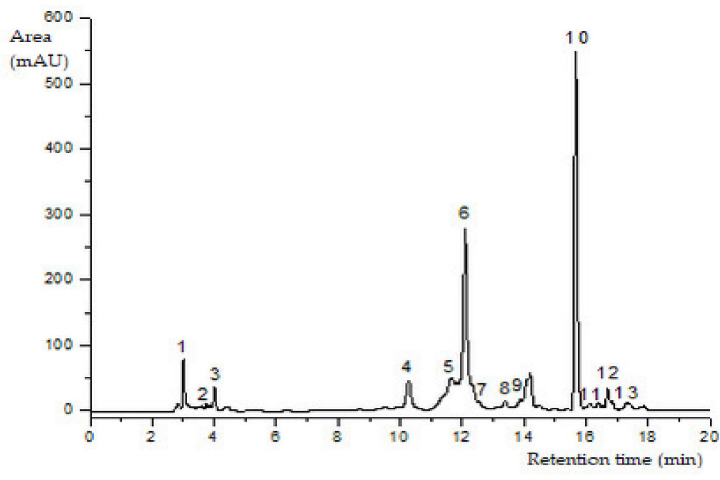

(c2). N5FrV $280 \mathrm{~nm}$

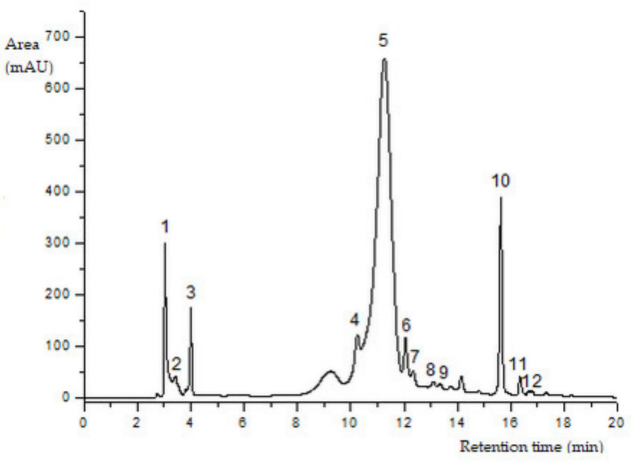

(e1). H7FrN $280 \mathrm{~nm}$

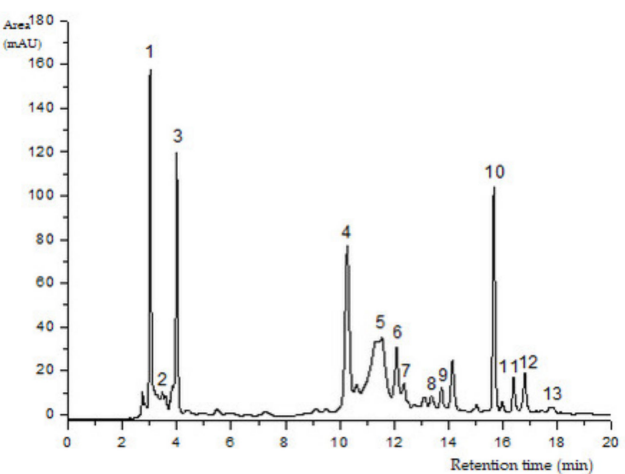

(d2). N6FrR $280 \mathrm{~nm}$

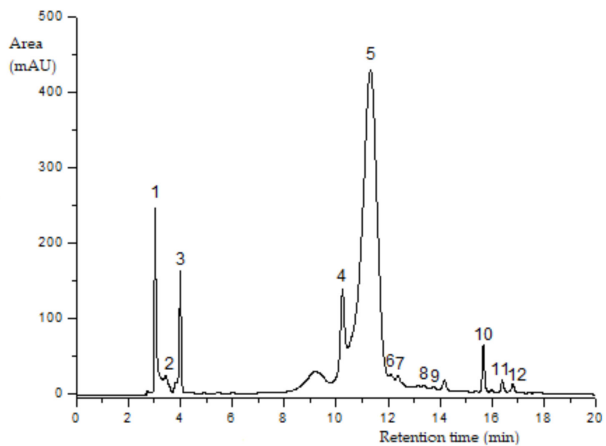

(e2). N7FrN $280 \mathrm{~nm}$

Figure 2. HPLC-ESI-MS chromatograms of the flavonoids content in elder samples (peak numbers correspond to those from Table 2).

Green elder flower buds (N1MV; H1MV) (with important chlorogenic acid values), blooming elder flowers (N4F and $\mathrm{H} 4 \mathrm{~F}$ ) (rich in rutin, kaempferol-3-rutinoside and procyanidin dimer isomer 3), and green unripe elderberries (N5FrV; H5FrV) (abundant in catechin, epicatechin and procyanidin dimer isomer 2) are the stages of major importance regarding the phenolic profile by HPLC of the studied elderberry varieties. Red unripe elderberries (N6FrR; H6FrR) do not show extreme values of flavonoid compounds; in contrast, purple-black elderberries (N7FrN; H7FrN) are abundant in cyanidin 3-sambubioside-5glucoside, cyanidin 3-sambubioside, cyanidin 3,5-diglucoside, procyanidin dimer isomer 1 and cyanidin 3-glucoside.

To better understand the results, PCA analysis (Figure 3) was performed for the 13 flavonoid compounds identified and quantified from Sambucus nigra L. and Sambucus nigra Haschberg, in different growth phases of the fruit (Table 2). 


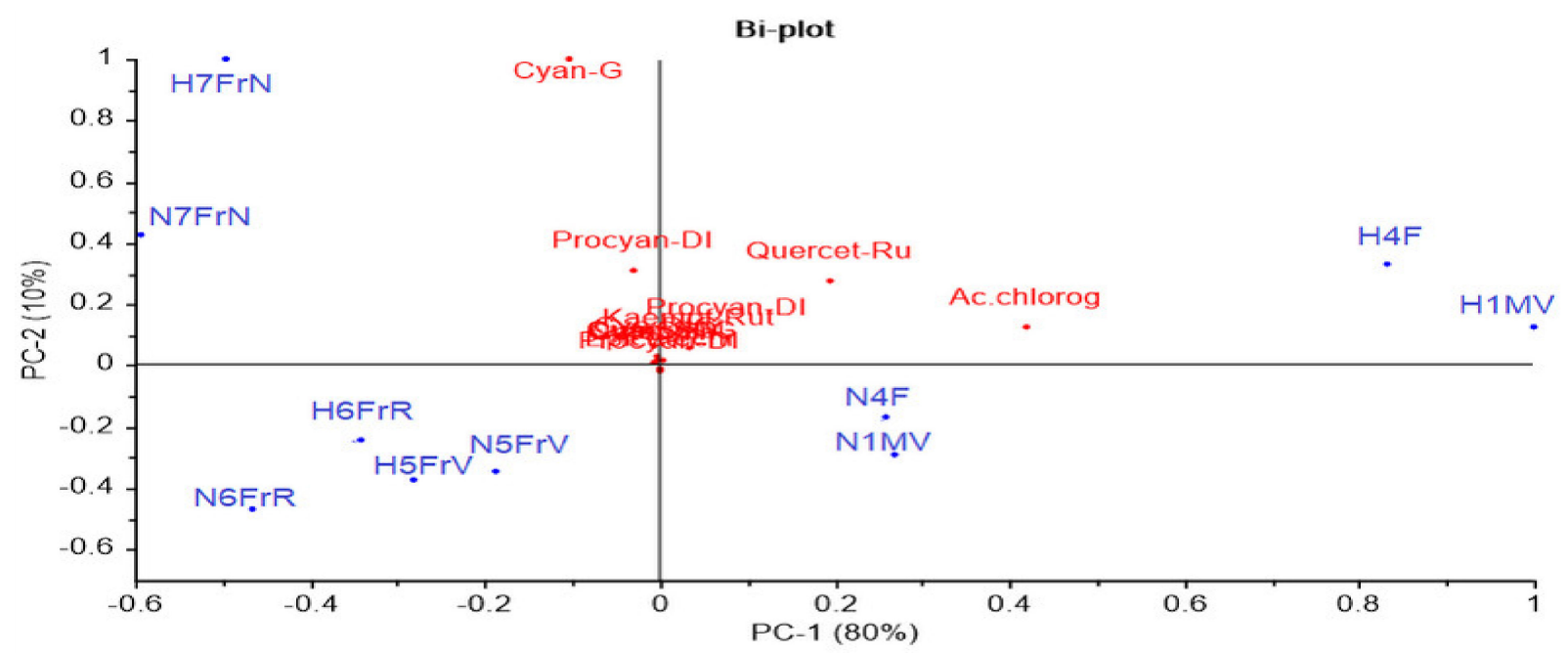

Figure 3. Principal component bi-plot analysis of 13 flavonoid variables measured by HPLC during ripening of elderberry. sam-5-gluc: cyanidin 3-sambubioside-5-glucoside; cy 3-sam: cyanidin 3-sambubioside; cy 3,5-digluc: cyanidin 3,5-diglucoside; procyan-DI: procyanidin dimer isomer1; cyan-G: cyanidin 3-glucoside; chlorog ac.: chlorogenic acid; catech: catechin; epicatech: epicatechin; procyan-D II: procyanidin dimer isomer 2; quercet-Ru: quercetin 3-rutinoside (rutin); q 3-gluc: quercetin 3-glucoside; k 3-rut: kaempferol-3-rutinoside; procyan-D III: procyanidin dimer isomer 3; anthocy: anthocyanins; flav: flavonols; hydroxyc ac: hydroxycinnamic acids.

\subsection{Mineral Content}

The analysis of the mineral content during the growth stages of the cultivated vs. wild elderberry shows extremely significant variations $(p \leq 0.001)$ in macroelements and microelements, noted in Table 3 . The highest value of minerals in the cultivated variety (Sambucus nigra Haschberg) is in the blooming stage (H4F), followed by buds (H1MV), green elderberries $(\mathrm{H} 5 \mathrm{FrV})$ and red elderberries $(\mathrm{H} 6 \mathrm{FrR})$. In the ripe fruit stage, the wild variety (Sambucus nigra L.) has higher mineral element values. 
Table 3. Macroelements and microelements of Transylvanian elderberry during seven ripening phases. Results are given as mean \pm SD.

\begin{tabular}{|c|c|c|c|c|c|c|c|c|c|c|c|c|}
\hline $\mathrm{Ca}$ & $\mathbf{K}$ & Mg & $\mathrm{Na}$ & Samples & $\mathrm{Fe}$ & $\mathrm{Cr}$ & $\mathrm{Cu}$ & Mn & Se & $\mathrm{Cd}$ & $\mathbf{P b}$ & $\mathrm{Ni}$ \\
\hline $298.336 \pm 0.004 \mathrm{~d}$ & $75.92 \pm 0.042 \mathrm{f}$ & $24.165 \pm 0.021 \mathrm{~b}$ & $28.045 \pm 0.007 \mathrm{i}$ & N1MV & $6.38 \pm 0.028 \mathrm{e}$ & $0.685 \pm 0.007 \mathrm{f}$ & $11.285 \pm 0.021 \mathrm{c}$ & $30.835 \pm 0.021 \mathrm{c}$ & $0.855 \pm 0.021 \mathrm{f}$ & $0.001 \pm 0.000 c$ & $0.004 \pm 0.000 \mathrm{~b}$ & $0.415 \pm 0.007 \mathrm{~g}$ \\
\hline $707.229 \pm 0.001 \mathrm{a}$ & $15.945 \pm 0.007 \mathrm{i}$ & $15.05 \pm 0.014 \mathrm{e}$ & $97.638 \pm 0.000 \mathrm{e}$ & H1MV & $1.185 \pm 0.007 \mathrm{~h}$ & $0.46 \pm 0.014 \mathrm{i}$ & $16.115 \pm 0.021 \mathrm{a}$ & $38.585 \pm 0.007 a$ & $0.915 \pm 0.007 \mathrm{e}$ & $0.002 \pm 0.000 \mathrm{~b}$ & $\mathrm{Nd}$ & $0.397 \pm 0.001 \mathrm{~h}$ \\
\hline $74.552 \pm 0.000 \mathrm{i}$ & $175.447 \pm 0.000 \mathrm{c}$ & $27.966 \pm 0.000 \mathrm{a}$ & $114.398 \pm 0.001 \mathrm{c}$ & $\mathrm{N} 4 \mathrm{~F}$ & $7.627 \pm 0.0001 \mathrm{~b}$ & $0.315 \pm 0.007 \mathrm{j}$ & $9.925 \pm 0.007 \mathrm{~d}$ & $25.21 \pm 0.000 \mathrm{~g}$ & $1.481 \pm 0.001 \mathrm{a}$ & $0.002 \pm 0.000 \mathrm{~b}$ & $0.001 \pm 0.000 \mathrm{~d}$ & $1.227 \pm 0.000 \mathrm{~d}$ \\
\hline $608.674 \pm 0.000 \mathrm{c}$ & $438.245 \pm 0.000 \mathrm{a}$ & $7.089 \pm 0.000 \mathrm{~g}$ & $12.727 \pm 0.000 \mathrm{j}$ & $\mathrm{H} 4 \mathrm{~F}$ & $8.52 \pm 0.000 \mathrm{a}$ & $3.142 \pm 0.003 \mathrm{a}$ & $6.891 \pm 0.001 \mathrm{~g}$ & $36.122 \pm 0.002 \mathrm{~b}$ & $0.562 \pm 0.002 \mathrm{~h}$ & $0.002 \pm 0.000 \mathrm{~b}$ & $0.005 \pm 0.000 \mathrm{a}$ & $0.252 \pm 0.000 \mathrm{i}$ \\
\hline $678.972 \pm 0.000 \mathrm{~b}$ & $21.506 \pm 0.000 \mathrm{~h}$ & $6.707 \pm 0.000 \mathrm{~h}$ & $72.726 \pm 0.000 \mathrm{~g}$ & H5FrV & $1.224 \pm 0.000 \mathrm{~g}$ & $0.89 \pm 0.000 \mathrm{~d}$ & $6.71 \pm 0.014 \mathrm{~h}$ & $30.174 \pm 0.005 \mathrm{~d}$ & $0.221 \pm 0.002 \mathrm{i}$ & $0.002 \pm 0.000 \mathrm{~b}$ & $\mathrm{Nd}$ & $1.475 \pm 0.000 \mathrm{~b}$ \\
\hline $215.874 \pm 0.000 \mathrm{~g}$ & $53.087 \pm 0.000 \mathrm{~g}$ & $22.687 \pm 0.000 \mathrm{c}$ & $141.004 \pm 0.000 \mathrm{~b}$ & N6FrR & $6.772 \pm 0.000 \mathrm{~d}$ & $2.78 \pm 0.000 \mathrm{~b}$ & $9.871 \pm 0.001 \mathrm{e}$ & $27.394 \pm 0.006 \mathrm{e}$ & $0.681 \pm 0.001 \mathrm{~g}$ & $0.002 \pm 0.000 \mathrm{~b}$ & $0.003 \pm 0.000 c$ & $0.421 \pm 0.000 \mathrm{~g}$ \\
\hline $235.743 \pm 0.000 \mathrm{f}$ & $257.261 \pm 0.000 \mathrm{~b}$ & $6.147 \pm 0.000 \mathrm{i}$ & $109.292 \pm 0.000 \mathrm{~d}$ & H6FrR & $0.963 \pm 0.000 \mathrm{i}$ & $0.63 \pm 0.000 \mathrm{~g}$ & $6.551 \pm 0.001 \mathrm{i}$ & $20.561 \pm 0.001 \mathrm{~h}$ & $1.15 \pm 0.000 \mathrm{c}$ & $0.002 \pm 0.000 \mathrm{~b}$ & $\mathrm{Nd}$ & $0.634 \pm 0.000 \mathrm{f}$ \\
\hline $156.476 \pm 0.000 \mathrm{~h}$ & $117.409 \pm 0.000 \mathrm{e}$ & $21.714 \pm 0.000 \mathrm{~d}$ & $57.344 \pm 0.000 \mathrm{~h}$ & N7FrN & $5.034 \pm 0.000 \mathrm{f}$ & $0.751 \pm 0.001 \mathrm{e}$ & $13.212 \pm 0.003 b$ & $13.87 \pm 0.000 \mathrm{j}$ & $0.705 \pm 0.007 \mathrm{~g}$ & $0.001 \pm 0.000 c$ & $0.001 \pm 0.000 \mathrm{~d}$ & $1.531 \pm 0.000 \mathrm{a}$ \\
\hline $71.239 \pm 0.000 \mathrm{j}$ & $152.187 \underset{* * *}{ \pm 0.000 \mathrm{~d}}$ & $2.618 \underset{* * *}{ \pm 0.000 \mathrm{j}}$ & $80.815 \pm 0.000 \mathrm{f}$ & H7FrN & $0.951 \pm 0.000 \mathrm{i}$ & $0.56 \pm \underset{* * *}{* 0.000 \mathrm{~h}}$ & $5.37 \pm 0.000 \mathrm{j}$ & $14.93 \pm 0.000 \mathrm{i}$ & $1.09 \pm 0.000 \mathrm{~d}$ & $0.003 \pm 0.000 \mathrm{a}$ & $\mathrm{Nd}$ & $0.909 \pm 0.000 \mathrm{e}$ \\
\hline *** & & & & (S) & & & $* * *$ & ${ }_{* * *}^{* * 4}$ & $F_{* * *}^{*}$ & ${ }_{* *}^{* *}$ & ${ }^{* * *}$ & ${ }^{* * *}$ \\
\hline
\end{tabular}

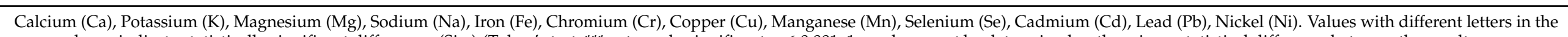
same column indicate statistically significant differences (Sig.) (Tukey's test, ${ }^{* * *}$ extremely significant $p \leq 0.001 ; 1-$ n.d., cannot be determined as there is no statistical difference between the results. 


\subsection{Water and Ash}

Water is an indispensable index in the quantification of technological maturity of Sambucus (Table 4).

Table 4. Percentage of humidity and ash determined in wild and cultivated elderberry (Sambucus nigra) genotypes during growth phases.

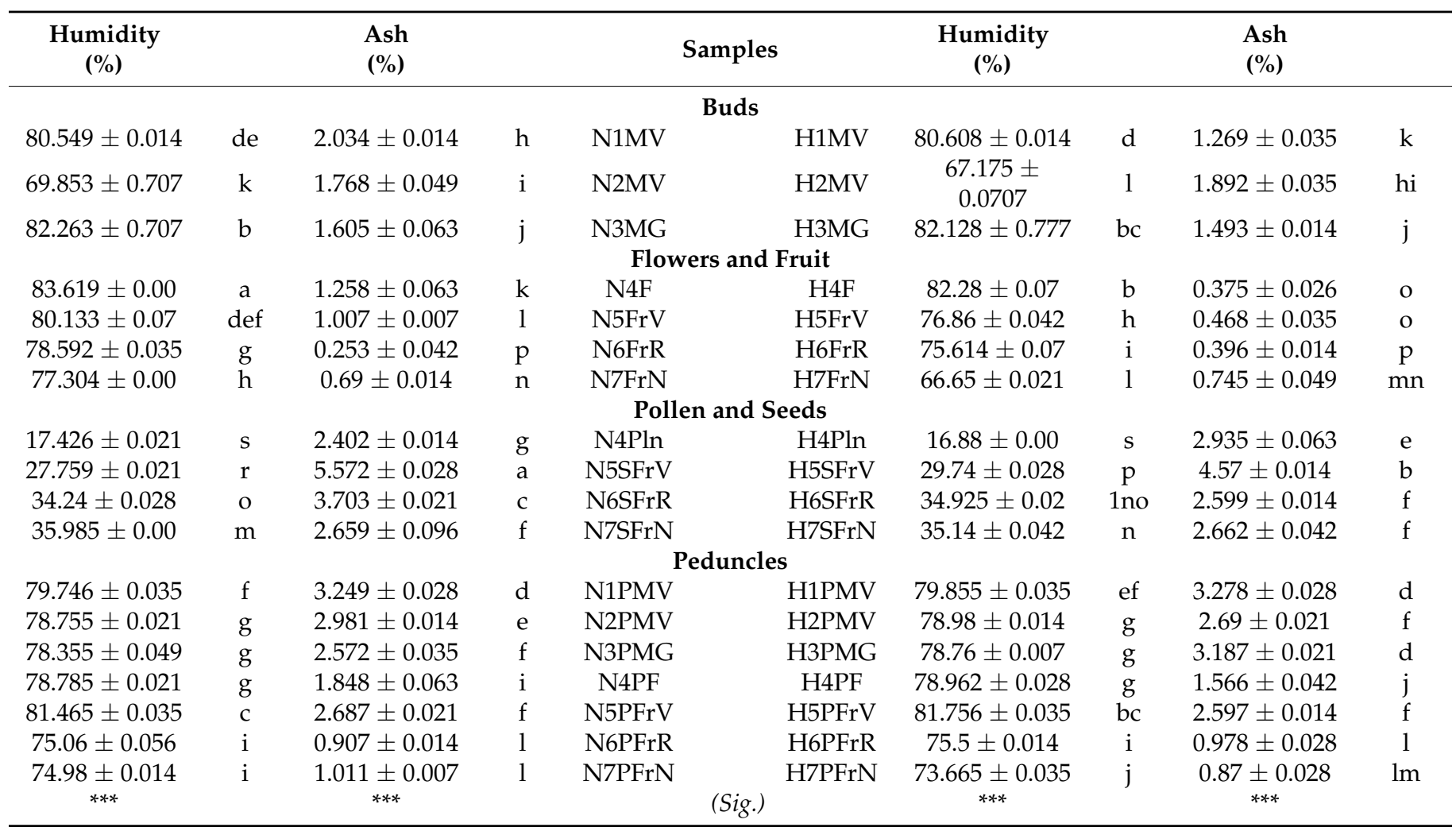

Values are expressed as mean. Values with different letters in the same column indicate statistically significant (Sig.) differences (Tukey's test, ${ }^{* * *}$ extremely significant $\left.p \leq 0.001\right)$.

\section{Discussion}

\subsection{Total Phenolic Content and Total Flavonoid Content}

TPC increases little after the ripe fruit stage has been attained, reaching for black elderberries $591.597 \pm 0.00 \mathrm{mg}$ GAE/100 g N7FrN and $679.254 \pm 0.004 \mathrm{mg}$ GAE/100 g H7FrN, which shows that the ripening process significantly influences the phenol content. Similarly to other dark colored fruits (Rubus L. and Vaccinium ashei Reade), the polyphenol content increases non-linearly with the ripening [31,32]. However, some studies [31] reported no significant differences, but these included green and red maturity of blackberry samples (Rubus L.) wildly grown in Turkey. Both in the wild S. nigra L. variety and in the Haschberg cultivar analyzed in the current study, there were significant differences in TPC due to the developmental stage of the samples and to the specificity of the two elderberry varieties analyzed.

The pollen grains in the Sambucus inflorescences are extremely rich in phenolic compounds (1201.126 $\pm 0.002 \mathrm{mg}$ GAE /100 g H4Pln), flavonoid content $(2100.23 \pm 0.001 \mathrm{mg}$ GAE/100 g H4Pln) and antioxidant activity ( $92.007 \pm 0.00 \% \mathrm{H} 4 \mathrm{Pln})$.

Pollen belonging to the wild or cultivated S. nigra variety is an important source of health beneficial compounds, suggesting that they could be useful in preventing diseases in which free radicals are involved. Such products decrease the risk of degenerative diseases by reducing oxidative stress and metal chelating activity, in addition to their reported anticarcinogenic properties [22]. The results obtained for pollen from elder flowers are 
comparable to those reported for Portuguese bee pollen, where similar TPC levels are found, but with a significantly higher flavonoid content [33].

The values of TPC in the samples of peduncles harvested during the elder flower bud phase have ascending dynamics up to a maximum value in the green elderberry bud peduncles (442.224 $\pm 0.00 \mathrm{mg}$ GAE/100 g N5PFrV and $429.963 \pm 0.002 \mathrm{mg}$ GAE/100 $\mathrm{g}$ for $\mathrm{H} 5 \mathrm{PFrV}$ ). During ripening of the elderberries, especially in the last maturity stages, the peduncles acquire a distinct reddish color, which is specific to the elder plant, suggesting the abundant presence of polyphenols.

During ripening, the TPC of the peduncle tends to increase from the red phase (N6PFrR and H6PFrR) to the dark color phase, and a similar tendency is seen for TPC values in the corresponding fruit. This is explained by the senescence of photosynthetic tissues in the peduncles [1]. Elderberry waste resulting from the elderberry processing industry (peduncles) could be an alternative source of unique bioactive natural polyphenols for other domains.

Additionally, in the context of using plant residues, studies indicate that seeds may contain significant levels of compounds with antioxidant and antiproliferative effects [10,34]. The total polyphenol content of red unripe elderberry seeds (57.614 $\pm 0.001 \mathrm{mg}$ GAE/100 g N6SFrR) was similar to that of the cultivated red unripe elderberry seeds (56.241 $\pm 0.007 \mathrm{mg}$ GAE/100 g H6SFrR).

The results showed a striking difference between the total polyphenol content in the seeds and ripe fruit of the Sambucus species (Table 2). TPC in the purple-black elderberry seeds (N7SFrN) was 10.07 lower compared to the edible purple-black ripe elderberries of the wild Sambucus species (N7FrN).

The literature also reports significant TPC contents in blackberry seeds (171.4 mg $\mathrm{GAE} / \mathrm{g}$ extract). In that case, total polyphenols in the seed residue extracts were strongly correlated with the antioxidant activity of those fruit, suggesting that phenolic compounds contribute to increasing the antioxidant capacity value [34].

The characterization of bioactive compounds in the Sambucus elderberry seeds during fructification, along with the presentation of their potential beneficial properties can lead to the added value use of these seeds and fruit. More precisely, the study contributes to increasing the profitability of elderberry fruit production and processing industries, as well as of seed oil manufacturers.

Previous studies have generally compared TPC values only for Sambucus nigra L. flower, fruit and leave samples; to our knowledge, so far, there have been no TPC reports for buds, peduncles or pollen. Sambucus nigra L. flowers contain even higher amounts of phenolic compounds compared to the fruit and leaves of this species [23]. Moreover, in the case of the current study, the variety and complexity (buds, peduncles, pollen) of the analyzed developmental phases of the elderberry (18 samples/variety) demonstrate the high degree of originality, indicating increased TPC values in white elder flower buds (883.066 $\pm 0.001 \mathrm{mg}$ GAE/100 g N3MG and 890.04 $\pm 0.00 \mathrm{H} 3 \mathrm{MG})$, peduncles (442.224 $\pm 0.00 \mathrm{mg} \mathrm{GAE} / 100 \mathrm{~g}$ and $429.963 \pm 0.002 \mathrm{mg}$ GAE $/ 100 \mathrm{~g} \mathrm{H}$ HPFrV) and pollen (1031.06 $\pm 0.014 \mathrm{mg}$ GAE/100 g N4Pln and $1201.126 \pm 0.002 \mathrm{mg}$ GAE/100 g H4Pln).

The TPC results obtained for the cultivated Haschberg elderberry varieties collected from areas with a predominantly temperate continental climate suggest slight differences. Thus, TPC values in the fruit of the Austrian Haschberg variety (364 mg GAE/100 g, $510 \mathrm{mg}$ GAE/100 g) [18] are lower than the values of the current study recorded for Haschberg in Romania (679.254 mg GAE/100 g H7FrN), and the wild flora samples (S. nigra) (591.597 $\pm 0.00 \mathrm{mg}$ GAE $/ 100 \mathrm{~g}$ N7FrN). In the fruit of the wild S. nigra L. variety in Slovenia (local population), TPC is $515 \mathrm{mg}$ GAE/100 g FW [19], a result similar to that obtained for wild growing elderberry in Romania (591.597 mg GAE/100 g FW). The Mediterranean elderberry culture area seems more favorable to the accumulation of phenolic compounds in elderberry flowers and fruit [13], registering higher values compared to the temperate zone ( $820 \mathrm{mg} \mathrm{GAE} / 100 \mathrm{~g}$ FW, $1177 \mathrm{mg}$ GAE/100 g FW, $1016 \mathrm{mg}$ $\mathrm{GAE} / 100 \mathrm{~g} \mathrm{FW}$ ). Compared to the variables of the present study (different growth stages 
and varieties of elderberry), in other publications TPC variability in fruit is established depending on altitude [24] or the effect of the harvesting year [13].

In conclusion, significant differences of TPC between the developmental stages were observed; mainly, a decrease from the maximum TPC value of white elder flower buds (N3MG and H3MG) to the fruit formation stages (N5FrV and H5FrV), followed by a slight increase in TPC in mature fruit (N5FrN and H5FrN) was recorded. The genotype proposed for medical applications is Haschberg genotype obtained in culture, a conclusion based on the high TPC values in pollen and fruit over the last developmental stages.

Total flavonoids mainly predominate in the white elder flower bud samples (1461.262 $\pm 0.038 \mathrm{mg}$ QE/100 g N3MG, $1369.17 \pm 0.084 \mathrm{mg}$ QE/100 g H3MG). Full blooming of the elder flower registers lower total flavonoid values $(973.178 \pm 0.001 \mathrm{mg}$ $\mathrm{QE} / 100 \mathrm{~g} \mathrm{~N} 4 \mathrm{~F}, 945.257 \pm 0.001 \mathrm{mg}$ QE/100 g H4F), but the pollen samples of both Sambucus varieties show maximum values $(2131.235 \pm 0.005 \mathrm{mg} Q \mathrm{QE} / 100 \mathrm{~g} \mathrm{~N} 4 \mathrm{Pl}, 2100.23 \pm 0.001$ $\mathrm{H} 4 \mathrm{Pl})$. Analyzing these high values of total flavonoids shows that white elder flower buds, as the complex organ of higher plants before the appearance of the flower, precede the maximum values of the flower pollen. During the full blooming phase, pollen in the elder flowers can be quantitatively diminished due to meteorological variations (wind, rain in particular), and this is also reflected in the lower value of total flavonoids.

By following the developmental stages of the elderberry, after blooming a decline in total flavonoid compounds was observed, during the formation of green elderberries (488.895 $\pm 0.002 \mathrm{mg} \mathrm{QE} / 100 \mathrm{~g} \mathrm{~N} 5 \mathrm{FrV}$ and $460.894 \pm 0.002 \mathrm{mg} \mathrm{QE} / 100 \mathrm{~g} \mathrm{H} 5 \mathrm{FrV})$, a decline that continues in the red elderberry stage $(304.548 \pm 0.01 \mathrm{mg}$ QE $/ 100 \mathrm{~g}$ N6FrR and $360.53 \pm 0.001 \mathrm{mg} \mathrm{QE} / 100 \mathrm{~g} \mathrm{H} 6 \mathrm{FrR})$. In fact, red elderberries have the lowest flavonoid compound value of the entire elderberry fructification process, in both analyzed varieties. Ripe elderberries (N7FrN and H7FrN) do not show considerable flavonoid compound accumulations, having only half of all flavonoids found in flowers (N4F and H4F). Compared to elderberries, elder flowers were classified as being richer in various phenolic compounds (especially flavonoids) [3,17]. The superiority of using flavonoid-rich natural extracts obtained from elder flowers over elderberries was highlighted by the qualitative and quantitative analysis (LC-ESI-MS/MS) of some corn snacks enriched with 5, 10 or $20 \%$ Sambucus nigra L. flower or fruit powder [15]. The results of the study above indicate that corn snacks with $20 \%$ elder flower addition represent an important source of natural antioxidants, and elder flowers can be excellent additions in obtaining functional foods.

Flavonoids can inhibit and promote iron absorption depending on their chemical structure due to chelation and/or reduction of iron [35]. However, despite the numerous studies regarding the therapeutic potential of the elderberry $[4,6,22]$, there is no information about the possible interactions with metals, particularly the two essential trace elements, iron and copper. Due to low iron chelating activity and high iron reducing activity under acidic conditions, elderberry fruit extracts might promote iron absorption in the duodenum or proximal jejunum due to the facilitated conversion of ferric ions to ferrous ions [35]. At the same time, this study indicates that Haschberg elderberry fruit extract was the strongest metal chelator, more effective compared to standards in all tested $\mathrm{pH}$ conditions, but its iron reducing properties were lower than standard ones.

A statistically insignificant moderate negative correlation ( $\mathrm{r}=-0.46, p=0.08 \mathrm{~ns})$ was achieved in our study using Pearson's correlation test (with a 95\% confidence interval) between flavonoids and iron content in samples N1MV, N4F, N5FrV, N6FrR, N7FrN, H1MV, H4F H5FrV, H6FrR and H7FrN.

Total flavonoid values in the seeds of both elderberry varieties varied similarly to those of the fruit from where they were separated. The red unripe seeds of both varieties (253.83 $\pm 0.012 \mathrm{mg}$ QE/100 g N6SFrR, $241.329 \pm 0.004 \mathrm{mg}$ QE/100 g H6SFrR) had, like in the case of red elderberries (N6FrR, H6FrR), the lowest total flavonoid values. Based on the variations recorded in the analyzed samples, one may suggest that flavonoid accumulation does not depend on genotype, but only on epigenetic factors. 
The interdependence between the peduncle and the pertaining buds, flowers or fruit, regarding the presence of total flavonoids, indicates a progressive increase with the developmental stages of the elderberry, up to the maximum limit recorded in the green elderberry peduncle $(986.828 \pm 0.00 \mathrm{mg}$ QE $/ 100 \mathrm{~g}$ N5PFrV and $900.654 \pm 0.00 \mathrm{mg}$ $\mathrm{QE} / 100 \mathrm{~g}$ H5PFrV). By comparing the green peduncle to the other analyzed samples, it can be seen that this might exceed even the elder flowers in terms of total flavonoid content $(973.178 \pm 0.001 \mathrm{mg}$ QE/100 N4F, $945.257 \pm 0.001 \mathrm{mg}$ QE/100 g H4F). The significant values of total flavonoids, total polyphenols $(442.224 \pm 0.00 \mathrm{mg} \mathrm{GAE} / 100 \mathrm{~g} \mathrm{~N} 5 \mathrm{PFrV}$ $429.963 \pm 0.002 \mathrm{mg} \mathrm{GAE} / 100 \mathrm{~g} \mathrm{H} 5 \mathrm{PFrV})$ and high antioxidant activity $(39.707 \pm 0.002 \%$ $\mathrm{N} 5 \mathrm{PFrV}, 42.565 \pm 0.004 \% \mathrm{H} 5 \mathrm{PFrV}$ ) obtained in the green elderberry peduncle make these plant parts alternative sources of unique bioactive natural compounds for various industries (agrifood industry, pharmaceutical industry, etc.).

In the fruit reddening period, red elderberry peduncles (N6PFrR and H6PFrR), ripe elderberry peduncles, respectively (N7PFrN and H7PFrN), had low total flavonoid compound values. It cannot be said that the fruit belonging to these peduncles followed the same decreasing trend of flavonoid values, because the ripe fruit (N7FrN and H7FrN) in both genotypes had increasing total flavonoid compound values towards the final maturity phase.

As expected, it was found that flavonoids were significantly positively correlated $(p \leq 0.05)$ with total polyphenols in the elder flowers, elderberries and peduncles $(r=0.679)$.

\subsection{Antioxidant Activity}

The most frequently associated key word with the Sambucus genus is antioxidant activity $[1,7,12,14,17,26]$. The mentioned authors indicate the antioxidant potential only in elderberry flowers and fruit (fresh, freeze-dried, pomace or powder samples), but across the developmental stages of the elderberry, according to the present study, other morphological parts with high antioxidant activity are observed (Table 1). Thus, it is important to find new sources of phytochemicals or new morphological parts of plants with high antioxidant activity (peduncles, pollen, buds) which can be used in food reformulation.

The antioxidant properties of various samples in the development of elderberry buds are attributed in the first place to the presence of anthocyanins and phenolic compounds. A directly proportional relationship was established between these compounds and DPPH antioxidant activity in the analyzed samples of both varieties. Antioxidant activity decreased from the pollen of wild and cultivated elder flowers $(91.007 \pm 0.000 \%$ N4Pln, $92.007 \pm 0.00 \% \mathrm{H} 4 \mathrm{Pln}$ ), to white elder flower buds (89.608 $\pm 0.001 \% \mathrm{~N} 3 \mathrm{MG}, 90.004 \pm 0.00 \%$ $\mathrm{H} 3 \mathrm{MG})$, to elder flowers $(87.01 \pm 0.002 \% \mathrm{~N} 4 \mathrm{~F}, 89.047 \pm 0.007 \% \mathrm{H} 4 \mathrm{~F})$ and green elder flower buds $(72.058 \pm 0.033 \% \mathrm{~N} 2 \mathrm{MG}, 70.057 \pm 0.002 \% \mathrm{H} 2 \mathrm{MG})$. Therefore, the primary developmental stages of the buds were well represented in the DPPHfree radical scavenging activity, which will increase until the appearance of elderberry inflorescences (N4F and $\mathrm{H} 4 \mathrm{~F}$ ). The current study justifies the abundance of antioxidants in elder flowers, which is reported in other studies [14,23], on account of maximum DPPH values recorded in the pollen of wild and cultivated elder flowers (N4Pln and H4Pln). However, recently, a study comparing elder flowers and fruit reported an equal or higher antioxidant capacity in elderberries. This is the only study highlighting the increased potential of fruit over flowers, motivating that the growth area and the analyzed cultivar can change the rule of accumulation in different morphological parts of the elderberry [14]. Thus, antioxidant capacity is significantly influenced by the analysis technique, the growth conditions and the individual variations of the analyzed plants. It should also be emphasized that the red and green fruit of the cultivated elderberry variety (H6FrR, H7FrV) showed a higher antioxidant activity than those harvested from the wild elderberry (Table 1). Regarding the cultivated variety, ripe Haschberg elderberries had the highest antioxidant capacity $(65.965 \pm 0.003 \%$ H7FrN) of all the analyzed fruit samples.

Statistically significant correlations were established $(p \leq 0.05)$ between DPPH free radical scavenging activity and chlorogenic acid values in the buds, flowers and fruit of the 
elderberry $(\mathrm{r}=0.711)$, as well as between flavonoid compounds $(\mathrm{r}=0.725)$, using Pearson's correlation test.

The high antioxidant capacity of the elderberry is related to the total polyphenol content (Table 1); many studies confirm the relationship between polyphenol content in elderberries and DPPH free radical scavenging $[7,12,14,36]$. Over the past years, Portuguese elderberry peduncles have been shown to obtain antioxidant capacity scores close to those of the pertaining ripe elderberries [1]. Among the analyzed peduncle samples, the similarity of DPPH values only occurs in the case of green elderberry peduncles of the wild variety (N5PFrV) and their fruit, respectively (N5FrV).

Elderberry seed methanolic extracts showed a reduced antioxidant capacity compared to peduncles. It can be observed, the scavenging effect of the Sambucus seed methanolic extract on DPPH free radicals increased with ripening: it varied from $7.723 \pm 0.002 \%$ N5SFrV to $9.012 \pm 0.012 \%$ N7SFrN. The same occurred in the case of seeds of the cultivated variety, $1 \%$ increases in DPPH free radical scavenging, between the developmental stages of elderberry seeds.

Comparable to other red fruits (chokeberry, blueberry or black currant), the antioxidant activity of elderberries ranks after the maximum values of Aronia fruit $(181 \mu \mathrm{mol}$ TE/g, DPPH method; $79 \mu \mathrm{mol} \mathrm{TE} / \mathrm{g}$, ABTS method), according to the study [36].

\subsection{HPLC-ESI-MS Characterization and Quantification of Phenolic Compositions}

The anthocyanin content in ripe elderberries was higher in the cultivated variety $(5235.606 \pm 0.002 \mu \mathrm{g} / \mathrm{mL}$ H7FrN) compared to the wild variety $(3545.226 \pm 0.008 \mu \mathrm{g} / \mathrm{mL}$ N7FrN) and 17 times higher than in the green fruit phenophase (in the cultivated elderberry $\mathrm{H} 5 \mathrm{FrV}$ ). Anthocyanin abundance in ripe elderberries is also mentioned by Kiprovski et al. [2] and Mikulic-Petkovsek et al. [19].

Cyanidin 3-sambubioside content starts at a green bud value of $33.788 \pm 0.002 \mu \mathrm{g} / \mathrm{ml}$ N1MV (it strictly occurs in the wild variety), disappears from the flower (both varieties $\mathrm{N} 4 \mathrm{~F}$ and $\mathrm{H} 4 \mathrm{~F}$ ), reappears in the green fruit (both varieties $\mathrm{N} 5 \mathrm{FrV}$ and $\mathrm{H} 5 \mathrm{FrV}$ ) and progressively increases with the ripening of elderberries (preponderantly higher content in the cultivated elderberry H6FrR and H7FrN). It should be noted that cyanidin 3-sambubioside and cyanidin 3-glucoside remain (they are not destroyed) in elderberry fruit-based preparations, except for fermented elderberry products [37]. Regarding cyanidin 3-sambubioside availability, this is significant even with low amounts [3].

The current study suggests that cyanidin 3-glucoside is the predominant pigment during the developmental stages of the elderberry. In the European Union, all colorants derived from anthocyanin are recognized as natural colorants in the E 163 classification. The interest in anthocyanic pigments has grown over the past years due to potential health benefits, including the reduction in the risk of coronary diseases, stroke, anticarcinogenic activity, anti-inflammatory effects, improved visual acuity and improved cognitive behavior [38].

The second important group of polyphenols includes flavonols, among which procyanidin dimer isomer 1 , catechin, epicatechin, procyanidin dimer isomer 2, quercetin 3rutinoside, quercetin 3-glucoside, kaempferol-3-rutinoside and procyanidin dimer isomer 3. The primary flavonol in the elder plant is rutin, while the other flavonols, quercetin 3 -glucoside and procyanidin dimer isomer 2, occur during ripening phases in smaller amounts.

The representative flavonols in the bud stage of the two studied varieties were kaempferol-3-rutinoside and procyanidin dimer isomer 3. Qercetin 3-rutinoside (rutin), quercetin 3-glucoside; kaempferol-3-rutinoside have higher values in the bud phase as well (N1MV and H1MV); procyanidin dimer isomer 3 increases in the blooming stage $(1827.588 \pm 0.002 \mu \mathrm{g} / \mathrm{mL}$ N4F and $2913.322 \pm 0.002 \mu \mathrm{g} / \mathrm{mL}$ H4F). The flowers of Haschberg cultivar (H4F) contain more phenolic compounds compared to the wild variety (N4F) and also compared to its fruit (H7FrN). 
Sambucus nigra Haschberg also continues to rank first regarding flavonoid compound accumulation in the green elderberry stage (H5FrV). Catechin $(396.994 \pm 0.002 \mu \mathrm{g} / \mathrm{mL})$, epicatechin $(205.707 \pm 0.002 \mu \mathrm{g} / \mathrm{mL})$ and procyanidin dimer isomer $2(239.426 \pm 0.002 \mu \mathrm{g} / \mathrm{mL})$ are predominant in green Haschberg elderberries $(\mathrm{H} 5 \mathrm{FrV})$.

Procyanidin dimer isomer 2, rutin, quercetin 3-glucoside, kaempferol-3-rutinoside, catechin contents become particular to the elderberry blooming phase, where an increase of each mentioned compound is followed by a decline during the subsequent fructification phase. The exception is catechin variation in the cultivated variety. The content of procyanidin dimer isomer 3 was quantified $2913.322 \pm 0.002 \mu \mathrm{g} / \mathrm{mL}$ in the flowers of Haschberg genotype. Procyanidins were the main flavonoids in the early developmental stages of the elderberry in both genotypes. During the elderberry fruit ripening stage, procyanidins were present only in the red unripe elderberry samples from the wild area (N6FrR). It was found that procyanidin dimer isomer 3 in the Haschberg variety decreased 27 times with ripening, reaching in green elderberries the value of $106.498 \pm 0.002 \mu \mathrm{g} / \mathrm{mL}$ H5FrV, compared to inflorescences $(\mathrm{H} 4 \mathrm{~F})$. Towards the end of the analyzed stages, procyanidin dimer isomer 3 in the cultivated variety became undetectable in the red (H6FrR) and purple-black elderberry phase (H7FrN) (Table 2). Additionally, in the wild S. nigra variety, the amount of procyanidins decreased to an undetectable level before anthocyanin synthesis. During the elderberry fruit maturity stage, the flavonoid content changes and each compound has its individual presence/disappearance pattern.

The phenolic profile during elder ripening by HPLC indicates the fact that red elderberries produce higher amounts of cinnamic acids and flavonol glycosides in fruit, particularly in the Haschberg variety. The cultivated variety is also higher in total phenolics compared to the wild genotype. In investigating hydroxycinnamic acids in the two genotypes, a linear correlation was found between total polyphenols, total flavonols, total hydroxycinnamic acids and antioxidant activity [36].

The main phenolic compound group during elder ripening was represented by hydroxycinnamic acids, the most important of which being chlorogenic acid. Other hydroxycinnamic acids found in Sambucus include, among others, neochlorogenic acid, cryptochlorogenic acid, 3- and 5-feruloylquinic acid and dicaffeoylquinic acids, including notably 1,5-di-caffeoylquinic acid [3] or caffeic, ferulic, p-coumaric acid [36]. The bioavailability profiles of chlorogenic acids suggest that they are metabolized mainly by the colonic microflora [39]; phenolic acids can be present as such in the majority of foods.

Chlorogenic acid was also detected in high amounts until close to the red fruit phase, when it showed a decline $(884.757 \pm 0.002 \mu \mathrm{g} / \mathrm{mL}$ H6FrR $)$. Similarly to the analysis of unripe elderberry fruit of Ljubostinja species, regarding chlorogenic acid, Kiprovski et al. also detect significant values [2]. The preparations of different elderberry products at $100{ }^{\circ} \mathrm{C}$ for a moderate length of time (less than $30 \mathrm{~min}$ ) showed an extreme chlorogenic acid decrease [37].

The two main components explained $90 \%$ of the total variance; however, no clear discrimination between the wild and the cultivated genotype was identified. As can be noticed in Figure 3, the developmental phases proved a higher influence as compared to cultivars; for example, the green buds (H1MV and N1MV) and flowers (H4F and N4F) from both cultivars registered high concentrations of quercetin 3-rutinoside and chlorogenic acid. In addition, purple-black fruit samples (H7FrN and N7FrN) showed the highest cyanidin 3-glucoside, procyanidin dimer isomer 1, cyanidin 3,5-diglucoside, cyanidin 3-sambubioside and cyanidin 3-sambubioside-5-glucoside concentrations.

Some differences in the flavonol and phenolic acid content can be explained by the variety, being closely related to the growth conditions, the degree of maturity, handling after storage or sample preparation treatments.

\subsection{Rutin as a Key Sambucus TPC during Growth Phases}

Quercetin 3-rutinoside (rutin) was the representative flavonol in the developmental stages of the studied elderberry varieties. In the Haschberg variety, the highest rutin value 
$(6178.463 \pm 0.002 \mu \mathrm{g} / \mathrm{mL} \mathrm{H4F})$, corresponding to the elder flower blooming stage, vas recorded. This rutin value confirms the contribution of the selection of the Sambucus genus to the detriment of the wild elderberry ( $>53 \%$ of total flavonol glycosides). Regardless of the measurement method, high rutin concentrations (approximately $50 \mathrm{mg} / \mathrm{g}$ per air-dried material) are found in Haschberg flores Sambuci in other studies as well [40]. The elder flower blooming stage becomes important because elder flower extracts contain important antioxidants, with potential benefits for human health [22]. However, the variety certainly influenced total rutin content, because both the flowers (H4F) and the buds of the cultivated elderberry (H1MV) had a higher rutin content than the wild variety.

During the development of the elder plant, the variation in rutin, for each elderberry variety, is due to its synthesis, more precisely enzymatic changes. Table 2 confirms that its synthesis starts in the green elder flower bud phase (N1MV and H1MV), suddenly increases in flowers (N4F and $\mathrm{H} 4 \mathrm{~F})$, registering a decline after the green elderberry phase (N5FrV and $\mathrm{H} 5 \mathrm{FrV})$. Rutin values were higher in all developmental stages of the fruit in the cultivated variety compared to the wild variety.

Green elderberries $(\mathrm{H} 5 \mathrm{FrV})$ contain twice as much rutin compared to ripe fruit $(\mathrm{H} 7 \mathrm{FrN})$, in the case of the cultivated variety. Rutin is not the predominant phenolic compound in ripe fruit (N7FrN; H7FrN). Authors such as Thomas et al. and Dominiquez et al. [12,41] describe ripe elderberries as being rich in rutin, without relating their value to the content of other developmental stages of the vegetation cycle of the elderberry shrub. More precisely, the rutin values mentioned in other publications for ripe fruit belonging to the Haschberg variety were $72.7 \mathrm{mg}$ rutin $100 \mathrm{~g}^{-1}$ [18] or $52.02 \pm 2.48 \mathrm{mg}$ CGE/100 $\mathrm{g}$ FW [42].

A parallel to the data obtained in the case of rabbiteye blueberry varieties cultivated in Guizhou (China) regarding phenolic compound accumulation during ripening shows that rutin tends to increase [32]. In contrast, rutin content decreases with the reddening of the fruit (N6FrR; H6FrR). According to the mentioned publications that study other plant organs of the elderberry shrub, such as the woody stems, green stems and leaves of Sambucus [2,43], rutin was also identified among the antioxidant compounds of the samples concerned. There is a currently increased interest in new plant extracts or unexploited plant parts, with a high flavonoid content. It was found that rutin is a source of biologically active phytochemicals, which can be used for the treatment of various disorders such as osteoporosis, hemorrhagic diseases, cardiovascular diseases, inflammation and cancer $[4,5]$. The previously mentioned studies also report that rutin has a significant pharmacological profile and could be used as a drug for the treatment of different types of dysfunctions.

In order to identify new natural sources of rutin, the green fruit of the cultivated elderberry (1504.402 $\pm 0.002 \mu \mathrm{g} / \mathrm{mL}, \mathrm{H} 5 \mathrm{FrV})$ can be an example, due to the high rutin values during the fructification stage (green, red and black). The stage of immature elderberries corresponding to the green color is followed by a marked decrease in rutin towards the stage of mature black ripe fruit $(\mathrm{H} 7 \mathrm{FrN})$. The concentration of this flavonol was lower in red or black elderberries than in unripe green fruit, probably due to the rapid conversion to anthocyanin, a compound that is accumulated during these stages. Similarly to the physiological mechanisms of flavonols found in Rubus sp. cousin. Loch Ness. in three maturation stages (green, red, black), flavonols decrease in the subsequent ripening stages [30]. The formation of anthocyanin is limited in many specific plant tissues and occurs during the explicit growth phase. The clear accumulation of these bioactive compounds usually provides clues about the biosynthetic catalysts running in the pathway. Following the phenylpropanoid pathway, anthocyanins can be synthesized [30]. Biosynthesis of anthocyanins requires both genes that directly participate in their formation and genes that control the transcription of the first genes.

Starting from the dispute in the biochemistry literature that an aglycon and its associated glycoside would act similarly [44], rutin as a major compound in the flowers, buds or green fruit might act pharmacodynamically with health promoting properties.

A rutin-rich $S$. nigra L. extract ( $463.20 \pm 0.00 \mu \mathrm{g} / \mathrm{mL})$, administered daily in $1 \mathrm{~mL}$ volume in vivo, proved to replace the activity level for superoxide dismutase (SOD) and 
catalase (CAT) at a value comparable to that of the control group; most of the anthocyanins in the plant extract were produced before penetration into the blood [22]. Rutin was also used as an ingredient for the treatment of diabetes and obesity, because it blocks the capillary walls, increases capillary flexibility and anticipates red blood cell and plasma infiltration outside the vessels, all of which due to its antioxidant property [17]. Rutin has an excellent potential even through its conversion to isoquercitin. Recently, its connection to the most widely used biodegradable and renewable thermoplastic polyester (PLA) was tested [45]. PLA is one of the biopolymers that has gained high interest with the aim of replacing polymers from inexhaustible sources. That study showed the fact that stabilizing polyesters by adding plant flavonoids significantly increased the oxidation resistance of both polylactide and polyhydroxyalkaonate.

Rutin was the major compound during the industrial processing of elderberry fruit as well. Rutin identified in S. nigra Vojsko products (Slovenia) (liqueur, juice, tea or elderberry jam) presented values from 2 to $65 \mathrm{mg} / \mathrm{kg}$, according to Senica et al. [37]. In the same line of thought, Mlynarczyk et al. [3] reported that in elderberry pomace, phenolic compounds, rutin in particular, represent the major component $(6-14 \mathrm{mg} / \mathrm{g} \mathrm{DW})$.

Furthermore, a recent study focusing on leaves and unripe fruit of the wild flora (from Fruškka Gora mountain, Serbia) and cultivated elderberry genotypes reveals the fact that the most important polyphenolic compounds are anthocyanins, with the predominance of quercetin 3-rutinoside (1-3\% DW in leaves and 0.5-0.8\% DW in fruit) [2].

Together, these approaches demonstrate that rutin in the structure of various parts of the Sambucus shrub is a key natural compound, a viable alternative to synthetic rutin. Flavonoids found in plant tissues represent in fact a secondary antioxidant defense system due to different stresses. Lipid peroxidation can be the result of oxidative stress that affects the integrity of the plant cell membrane. In this sense, rutin interacts with the polar head of phospholipids at the lipid water interface, increasing membrane rigidity and, consequently, protects the membranes against oxidative deterioration [44]. The relation of the rutin structure function is the representation of major biological activities.

Consequently, the flowers (H4F) and green buds (H1MV) of the Haschberg variety had the best bioactive properties of the studied samples, particularly due to the presence of rutin. Therefore, new identification of plant sources of rutin can take the form of promising dietary supplements.

\subsection{Mineral Content}

There are no available data in the literature regarding the profile of minerals during the development of the elderberry; however, the elderberry fruit, flowers, leaves or stems have been distinctly characterized [14,46]. The biological material in the samples of the early developmental stages of the elderberry (N1MV, H1MV, N4F and H4F) is the richest in macronutrients $(\mathrm{Ca}, \mathrm{K}, \mathrm{Mg}$ and $\mathrm{Na}$ ), particularly in green fruit, having a high Na content $(\mathrm{N} 5 \mathrm{FrV})$. Thus, the buds of the cultivated elderberry are extremely rich in Ca $(707.229 \pm 0.001 \mathrm{mg} / \mathrm{kg} \mathrm{H1MV})$, the flowers of the wild elderberry are rich in $\mathrm{K}$ $(438.245 \pm 0.000 \mathrm{mg} / \mathrm{kg} \mathrm{N} 4 \mathrm{~F})$ and $\mathrm{Mg}(27.966 \pm 0.000 \mathrm{mg} / \mathrm{kg} \mathrm{H} 4 \mathrm{~F})$, and green fruit are rich in $\mathrm{Na}(209.725 \pm 0.000 \mathrm{mg} / \mathrm{kg} \mathrm{N5FrV})$.

There is an extremely significant variation of calcium $(p \leq 0.001)$ in the Haschberg variety, which reaches the maximum value in the green elder flower bud phase (H1MV), also having high values in flowers $(\mathrm{H} 4 \mathrm{~F})$ and green fruit $(\mathrm{H} 5 \mathrm{FrV})$. For the same elderberry variety, Młynarczyk et al. [14] focused on the idea that flowers are richer in calcium $(2724.7 \mu \mathrm{g} / \mathrm{g}$ dry weight) compared to the fruit of the plant. Therefore, in the flower development phase, calcium was present in high values, according to the mentioned study, but the current research presents significant values starting with elder flower buds. Detected in the vacuoles and the cell wall of organic matter, calcium fulfills various functions in the secretory and structural processes of tissues. Consequently, in the present study, after the blooming phase (H4F), high calcium values in the cell walls of unripe green elderber- 
ries $(\mathrm{H} 5 \mathrm{FrV})$ are maintained, but with the cell wall softening in the fruit maturity phase (H7FrN), a decline occurs due to the different way of calcium binding in tissue.

Ripe fruit contain less calcium than green fruit; this is explained by the messenger function of calcium in cellular signal transduction, which involves accumulation in the initial developmental stages of the elderberry [46].

During the growth stages of the analyzed samples, magnesium registers higher values in the case of the wild variety. Edible Sambucus nigra L. flowers contain the highest concentration of $\mathrm{Mg}(27.966 \mathrm{mg} / \mathrm{kg})$ and Se $(1.481 \mathrm{mg} / \mathrm{kg})$, while edible Haschberg flowers contain the highest concentration of K $(438.245 \mathrm{mg} / \mathrm{kg}), \mathrm{Fe}(8.52 \mathrm{mg} / \mathrm{kg})$ and $\mathrm{Cr}(3.142 \mathrm{mg} / \mathrm{kg})$.

The recommendations of Dietary Reference Intakes (DRIs), according to Regulation (EU)No1169/2011 [47] are as follows for an adult: magnesium (375 mg/day), selenium

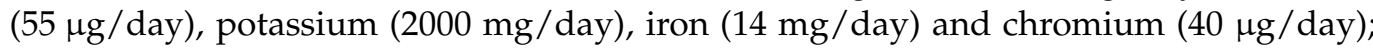
based on this, edible elder flowers (regardless of the form of growing) can represent acceptable organic sources for the daily mineral requirements.

The variations in $\mathrm{Mg}$ content in the samples included in the study can be explained by the implication of magnesium in the middle of the porphyrin ring attributed to the primary stage of chlorophyll biosynthesis and in carbohydrate partitioning metabolism. The introduction of magnesium into the structure of porphyrin is the first step of chlorophyll biosynthesis. Major changes during the development of the elderberry have been observed for magnesium, which is the central atom of the chlorophyll molecule, along with iron and copper, ensuring chlorophyll synthesis functionality.

Having a large bioavailability in plants and being easy to transport to the constitutive parts of plant organs, sodium was identified 7.4 times more in green elderberries (N5FrV) than in green elder flower buds in the wild variety (N1MV). The variation of sodium in the analyzed samples ranges between $12.727-209.725 \mathrm{mg} / \mathrm{kg}$. Compared to the study of some medicinal plants, sodium can have values from $37-5584 \mathrm{mg} / \mathrm{kg} \mathrm{[46].}$

Potassium in both varieties registers a considerable decrease after the blooming phase (N4F and H4F), followed by an ascending accumulation tendency.

The influence of the growth stages on the microelements identified ( $\mathrm{Fe}, \mathrm{Cr}, \mathrm{Cu}, \mathrm{Mn}, \mathrm{Se}$, $\mathrm{Cd}, \mathrm{Pb}$ and $\mathrm{Ni}$ ) indicates maximum values in the primary growth stages of the elderberry, like in the case of macronutrients. Only the metals $\mathrm{Cd}$ and $\mathrm{Ni}$ do not obey this rule, being predominant in the ripe fruit phase (N7FrN and H7FrN). The daily recommended amounts are small for micronutrients $\mathrm{Fe}, \mathrm{Cu}$ and $\mathrm{Mn}$ (less than $100 \mathrm{mg}$ /day), but the dietary plant sources rich in such micronutrients are important. This is explained by their involvement in the regulation of the nervous system function [46].

The growth stages of Sambucus have an impact on iron content, which is lower in the case of the cultivated genotype compared to the wild genotype, across all developmental stages (from buds to ripening).

The mineral elements $\mathrm{Fe}, \mathrm{Mn}, \mathrm{Cu}$ are actively involved in photosynthesis, in redox processes, $\mathrm{Cu}$ being a component of lignified tissue, also participating in pollen formation.

While copper, manganese and cadmium content have similar values in the different stages, nickel, lead, selenium, chromium and iron show oscillations during the ripening period (Table 3), in both varieties.

Chromium and manganese concentrations increased in the elderberry reddening period (N6FrR and H6FrR) and decreased in the maturity stage (N7FrN and H7FrN). Cadmium remained constant during elderberry development. The amount of accumulated heavy metals $(\mathrm{Fe}, \mathrm{Cr}, \mathrm{Cu}, \mathrm{Cd}, \mathrm{Pb}$ and $\mathrm{Ni}$ ) is dependent on the development phase only in the case of the cultivated elderberry variety. Green Haschberg buds (H1MV) contain almost twice as much heavy metals as red fruit (H6FrR).

However, Haschberg variety accumulated few heavy metals in the analyzed metals, a probable consequence of the culture area. It should also be noted that the Haschberg variety orchard is located in a rural area (Manau village), far from the heavy road traffic, which has a significant impact on the chemical composition of the elderberry. Studies report a significant correlation between the heavy metal content in the elderberry fruit and 
flowers in Poland and the influence of road traffic routes [25]. The proximity and intensity of the road traffic significantly contribute to the increase in the content of these elements in the analyzed raw material.

Even if Sambucus Nigra Haschberg fructus contains the highest amount of Cd $(0.003 \mathrm{mg} / \mathrm{kg}$ H7FrN) and Sambucus nigra L. fructus has the highest Ni value (1.531 mg/kg N7FrN) of the elderberry growth stages, this does not prevent their optimal use in dietary or curative formulas. However, the values of heavy metals accumulated in the ripe fruit stage in both varieties do not exceed the limit of accepted concentrations $(0.30 \mathrm{mg} \mathrm{Cd} / \mathrm{kg}$ and $0.1-5 \mathrm{mg}$ $\mathrm{Ni} / \mathrm{kg}$ ), recognized by the World Health Organization (WHO) [46].

Mainly analyzed for their commercial use, elderberry fruit have variable amounts of Ca (574-1528 mg/kg FW), K (2953-5494 mg/kg FW) and Na (13-146 mg/kg FW) [3].

The fruit at full maturity had optimal catalytic equipment for functioning even outside the plant, but the plant organs which appear throughout the genetic ripening process show an increase in growth hormone biosynthesis. Hence, a well-defined mineral variation results (12 minerals identified).

Among the identified minerals, cadmium was the heavy metal with the lowest values during the elderberry fructification period in both varieties. Lead was undetected in four growth stages in the cultivated variety (buds, green, red and ripe fruit).

In conclusion, the macronutrients and micronutrients predominating in different developmental stages of the studied varieties were: in green elder flower buds, calcium, copper and magnesium to an equal extent; in elder flowers, potassium and magnesium, iron, chromium and selenium. In green elderberries, the highest value of macronutrients was given by sodium, and among heavy metals, lead was well represented. In the case of red elderberries, the variation of microelements and macroelements did not reach a maximum value; in ripe elderberries, nickel was well represented. Therefore, the ripening phases highlight a new promising source of mineral elements in human nutrition.

Consequently, the individual synthesis of minerals is better represented in the cultivated variety than in the wild one. Thus, a good adaptation of the Haschberg variety to the conditions of the temperate zone and the implicit selection of the variety are observed.

\subsection{Water and Ash}

Humidity registered the lowest values in the pollen sample of the cultivated variety (16.880\% H4Pln) and the highest values in wild elderberry inflorescences (83.61\% N4F). In the case of elderberry fruit (N7FrN and H7FrN), humidity percentages can be comparable to those reported by $\mathrm{Wu}$ et al. [24].

Total ash content in the analyzed elderberry samples was extremely significant ( $p \leq 0.001$ ), dependent on the developmental phases (Table 4). Until the full blooming stage of the elderberry, ash had the highest values in pollen samples (2.93\% HPln). Studies report that $S$. nigra L. inflorescences contain considerable amounts of total ash [14], being directly dependent on the amount of pollen in the constitution of the flower. The ash of green elderberry seeds (N5FrV, $\mathrm{H} 5 \mathrm{FrV})$, regardless of the site of growth, showed maximum values (about $5 \%$ of total ash content), indicating the fact that Transylvanian elderberry seeds can be considered valuable organic reservoirs of mineral elements. Not only the peduncle color) [1], but also total ash values change in the last developmental stage of the elderberry. In the peduncle/flower, mature/immature fruit relationship, the percentage of ash was higher in peduncles. From a biochemical point of view, elderberry flowers had a high-water content but flower buds had a higher total ash percentage.

\section{Summary and Conclusions}

The developmental stages of the elderberry have an extremely significant impact $(p \leq 0.001)$ on the various bioactive compounds identified and implicitly on antioxidant properties. TPC and antioxidant activity vary across the developmental stages of elderberries: in the early phase, green buds have high values that increase during the evolution of the bud, until before the flower onset. The elder flower blooming stage announces a 
decline in bioactive compounds in the elderberry; the decrease continues in the green and red elderberry stage, and there is a slight increase towards the full maturity of the fruit.

The pollen samples in the inflorescences of both Sambucus varieties were extremely rich in phenolic compounds, flavonoids, and had a high antioxidant action. Green elder flower buds contained significant amounts of chlorogenic acid, calcium, copper and magnesium. White elder flower buds represent the key developmental stage, due to the high values of total flavonoids, exceeding by far those of elderberry inflorescences. However, the elder flower blooming stage is important due to the total content of rutin, quercetin 3-glucoside, kaempferol-3-rutinoside; procyanidin dimer isomer 3; and among minerals, $\mathrm{K}$ and $\mathrm{Mg}$. The variety influences total rutin content, because both the flowers and the buds of the Haschberg variety have a higher content of rutin than the wild variety. Therefore, new possible organic sources of rutin can be taken into consideration by various fields that promote a healthy life. During ripening of elderberries, especially in the last maturity stages, the peduncles acquire a distinct reddish color, which is specific to the elder plant, suggesting the abundant presence of polyphenols. During ripening, the TPC of the peduncle tends to increase from the red phase to the dark color phase, similarly to TPC values in the corresponding fruit. This is explained by the senescence of photosynthetic tissues in the peduncles. By comparing the green peduncles to the other analyzed samples, it can be observed that these might exceed even the elder flowers in terms of total flavonoid content. Peduncles represent the key biological material in this study, alongside elderberry seeds, through their mineral content as well. Green elderberries abound in catechin, epicatechin, procyanidin dimer isomer 2 and sodium. Red elderberries have moderate or low values of the studied compounds of the future ripe fruit. Ripe elderberries registered the highest concentrations of cyanidin 3-glucoside, procyanidin dimer isomer 1, cyanidin 3,5-diglucoside, cyanidin 3-sambubioside and cyanidin 3-sambubioside-5-glucoside.

The genotype proposed for medical applications is Haschberg genotype obtained in culture, a conclusion based on the high TPC values of pollen and fruit in the last developmental stages, the highest rutin concentrations in flowers, the calcium content in green buds, and $\mathrm{K}$ and Fe content in flowers.

This knowledge significantly contributes to creating the profile of bioactive compounds of the new natural resources of the common elderberry shrub from buds to ripening. The composition of vegetative stay of Sambucus recommends it as an important factor for nutrition and health.

Author Contributions: Conceptualization, S.M. and G.S.M.; methodology, A.E.M., V.M. and C.C.M.; software, C.R.P.; validation, V.M., R.A.U.; writing—original draft preparation, G.S.M.; writingreview and editing, R.A.U. and R.M.M.; visualization, G.B.; supervision, S.M.; project administration, G.S.M., V.M. and R.A.U. All authors have read and agreed to the published version of the manuscript.

Funding: This research received no external funding.

Institutional Review Board Statement: Not applicable.

Informed Consent Statement: Not applicable.

Data Availability Statement: The data presented in this study are available on request from the corresponding author, due to privacy restriction.

Acknowledgments: This work was supported by a grant of the Romanian Ministry of Education and Research, CCCDI-UEFISCDI, project number PN-III-P2-2.1-PED-2019-5346.

Conflicts of Interest: The authors declare no conflict of interest.

\section{References}

1. Silva, P.; Ferreira, S.; Nunes, F.M. Elderberry (Sambucus nigra L.) by-products a source of anthocyanins and antioxidant polyphenols. Ind. Crop. Prod. 2017, 95, 227-234. [CrossRef]

2. Kiprovski, B.; Malenčić, Đ.; Ljubojević, M.; Ognjanov, V.; Veberic, R.; Hudina, M.; Mikulic-Petkovsek, M. Quality parameters change during ripening in leaves and fruits of wild growing and cultivated elderberry (Sambucus nigra) genotypes. Sci. Hortic. 2021, 277. [CrossRef] 
3. Mlynarczyk, K.; Walkowiak-Tomczak, D.; Lysiak, G.P. Bioactive properties of Sambucus nigra L. as a functional ingredient for food and pharmaceutical industry. J. Funct. Foods 2018, 40, 377-390. [CrossRef]

4. Stănciuc, N.; Oancea, A.M.; Aprodu, I.; Turturică, M.; Barbu, V.; Ioniţă, E.; Râpeanu, G.; Bahrim, G. Investigations on binding mechanism of bioactives from elderberry (Sambucus nigra L.) by whey proteins for efficient microencapsulation. J. Food Eng. 2018, 223, 197-207. [CrossRef]

5. Harnett, J.; Oakes, K.; Care, J.; Leach, M.; Brown, D.; Cramer, H.; Pinder, T.A.; Steel, A.; Anheyer, D. The effects of Sambucus nigra berry on acute respiratory viral infections: A rapid review of clinical studies. Adv. Integr. Med. 2020, 7, 240-246. [CrossRef]

6. Weng, J.-R.; Lin, C.-S.; Lai, H.-C.; Lin, Y.-P.; Wang, C.-Y.; Tsai, Y.-C.; Wu, K.-C.; Huang, S.-H.; Lin, C.-W. Antiviral activity of Sambucus FormosanaNakai ethanol extract and related phenolic acid constituents against human coronavirus NL63. Virus Res. 2019, 273. [CrossRef]

7. Mikulic-Petkovsek, M.; Ivancic, A.; Schmitzer, V.; Veberic, R.; Stampar, F. Comparison of major taste compounds and antioxidative properties of fruits and flowers of different Sambucus species and interspecific hybrids. Food Chem. 2016, 200, 134-140. [CrossRef]

8. Wrońska-Pilarek, D.; Jagodziński, A.M.; Bocianowski, J.; Marecik, M.; Janyszek-Sołtysiak, M. Pollen morphology and variability of Sambucus nigra L.-Adoxaceae. Biologia 2020, 75, 481-493. [CrossRef]

9. Salvador, A.C.; Silvestre, A.J.; Rocha, S.M. Unveiling elderflowers (Sambucus nigra L.) volatile terpenic and norisoprenoids profile: Effects of different postharvest conditions. Food Chem. 2017, 229, 276-285. [CrossRef]

10. Dulf, F.; Oroian, I.; Vodnar, D.; Socaciu, C.; Pintea, A. Lipid Classes and Fatty Acid Regiodistribution in Triacylglycerols of Seed Oils of Two Sambucus Species (S. nigra L. and S. ebulus L.). Molecules 2013, 18, 11768-11782. [CrossRef]

11. Salvador, A.C.; Rudnitskaya, A.; Silvestre, A.J.; Rocha, S.M. Metabolomic-Based Strategy for Fingerprinting of Sambucus nigra L. Berry Volatile Terpenoids and Norisoprenoids: Influence of Ripening and Cultivar. J. Agric. Food Chem. 2016, 64, 5428-5438. [CrossRef] [PubMed]

12. Dominguez, R.; Zhang, L.; Rocchetti, G.; Lucini, L.; Pateiro, M.; Munekata, P.E.S.; Lorenzo, J.M. Elderberry (Sambucus nigra L.) as potential source of antioxidants. Characterization, optimization of extraction parameters and bioactive properties. Food Chem. 2020, 330, 127266. [CrossRef]

13. Ferreira, S.S.; Silva, P.; Silva, A.M.; Nunes, F.M. Effect of harvesting year and elderberry cultivar on the chemical composition and potential bioactivity: A three-year study. Food Chem. 2020, 302, 125366. [CrossRef]

14. Mlynarczyk, K.; Walkowiak-Tomczak, D.; Staniek, H.; Kidon, M.; Lysiak, G.P. The Content of Selected Minerals, Bioactive Compounds, and the Antioxidant Properties of the Flowers and Fruit of Selected Cultivars and Wildly Growing Plants of Sambucus nigra L. Molecules 2020, 25, 876. [CrossRef] [PubMed]

15. Oniszczuk, A.; Olech, M.; Oniszczuk, T.; Wojtunik-Kulesza, K.; Wójtowicz, A. Extraction methods, LC-ESI-MS/MS analysis of phenolic compounds and antiradical properties of functional food enriched with elderberry flowers or fruits. Arab. J. Chem. 2019, 12, 4719-4730. [CrossRef]

16. Lorenzo, J.M.; Pateiro, M.; Dominguez, R.; Barba, F.J.; Putnik, P.; Kovacevic, D.B.; Shpigelman, A.; Granato, D.; Franco, D. Berries extracts as natural antioxidants in meat products: A review. Food Res. Int. 2018, 106, 1095-1104. [CrossRef]

17. Sidor, A.; Gramza-Michałowska, A. Advanced research on the antioxidant and health benefit of elderberry (Sambucus nigra) in food-A review. J. Funct. Foods 2015, 18, 941-958. [CrossRef]

18. Lee, J.; Finn, C.E. Anthocyanins and other polyphenolics in American elderberry (Sambucus canadensis) and European elderberry (S. nigra) cultivars. J. Sci. Food Agric. 2007, 87, 2665-2675. [CrossRef] [PubMed]

19. Mikulic-Petkovsek, M.; Schmitzer, V.; Slatnar, A.; Todorovic, B.; Veberic, R.; Stampar, F.; Ivancic, A. Investigation of anthocyanin profile of four elderberry species and interspecific hybrids. J. Agric. Food Chem. 2014, 62, 5573-5580. [CrossRef]

20. Sytar, O.; Kosyan, A.; Taran, N.; Smetanska, I. Anthocyanin's as marker for selection of buckwheat plants with high rutin content. Gesunde Pflanz. 2014, 66, 165-169. [CrossRef]

21. Enogieru, A.B.; Haylett, W.; Hiss, D.C.; Bardien, S.; Ekpo, O.E. Rutin as a Potent Antioxidant: Implications for Neurodegenerative Disorders. Oxid. Med. Cell. Longev. 2018, 2018, 6241017. [CrossRef]

22. Ungur, R.; Buzatu, R.; Lacatus, R.; Purdoiu, R.C.; Petrut, G.; Codea, R.; Sarpataky, O.; Biris, A.; Popovici, C.; Mircean, M.; et al. Evaluation of the Nephroprotective Effect of Sambucus Nigra Total Extract in a Rat Experimental Model of Gentamicine Nephrotoxicity. Rev. Chim. 2019, 6, 1971-1974. [CrossRef]

23. Dawidowicz, A.L.; Wianowska, D.; Baraniak, B. The antioxidant properties of alcoholic extracts from Sambucus nigra L. (antioxidant properties of extracts). LWT Food Sci. Technol. 2006, 39, 308-315. [CrossRef]

24. $\mathrm{Wu}, \mathrm{X}$.; Gu, L.; Prior, R.L.; Mckay, S. Characterization of Anthocyanins and Proanthocyanidins in Some Cultivars of Ribes, Aronia, and Sambucus and Their Antioxidant Capacity. J. Agric. Food Chem. 2004, 52, 7846-7856. [CrossRef]

25. Kołodziej, B.; Antonkiewicz, J.; Maksymiec, N.; Drożdżal, K. Effect of traffic pollution on chemical composition of raw elderberry (Sambucus nigra L.). J. Elemntology 2012, 17, 67-78. [CrossRef]

26. Duymus, H.G.; Goger, F.; Baser, K.H. In vitro antioxidant properties and anthocyanin compositions of elderberry extracts. Food Chem. 2014, 155, 112-119. [CrossRef]

27. Diaconeasa, Z.; Leopold, L.; Rugina, D.; Ayvaz, H.; Socaciu, C. Antiproliferative and antioxidant properties of anthocyanin rich extracts from blueberry and blackcurrant juice. Int. J. Mol. Sci. 2015, 16, 2352-2365. [CrossRef] 
28. Bunea, A.; Rugina, D.; Sconta, Z.; Pop, R.M.; Pintea, A.; Socaciu, C.; Tabaran, F.; Grootaert, C.; Struijs, K.; VanCamp, J. Anthocyanin determination in blueberry extracts from various cultivars and their antiproliferative and apoptotic properties in B16-F10 metastatic murine melanoma cells. Phytochemistry 2013, 95, 436-444. [CrossRef]

29. Pasca, C.; Marghitas, L.; Dezmirean, D.; Bobis, O.; Bonta, V.; Chirila, F.; Matei, I.; Fit, N. Medicinal Plants Based Products Tested on Pathogens Isolated from Mastitis Milk. Molecules 2017, 22, 1473. [CrossRef]

30. Garcia-Seco, D.; Zhang, Y.; Gutierrez-Manero, F.J.; Martin, C.; Ramos-Solano, B. Application of Pseudomonas fluorescens to Blackberry under Field Conditions Improves Fruit Quality by Modifying Flavonoid Metabolism. PLoS ONE 2015, 10, e0142639. [CrossRef]

31. Ilkay, T.; Sule, U.N.; Belkis, T. Physical and chemical changes during ripening of blackberry fruits. Sci. Agric. 2008, 65, 87-90.

32. Guofang, X.; Xiaoyan, X.; Xiaoli, Z.; Yongling, L.; Zhibing, Z. Changes in phenolic profiles and antioxidant activity in rabbiteye blueberries during ripening. Int. J. Food Prop. 2019, 22, 320-329. [CrossRef]

33. Morais, M.; Moreira, L.; Feas, X.; Estevinho, L.M. Honeybee-collected pollen from five Portuguese Natural Parks: Palynological origin, phenolic content, antioxidant properties and antimicrobial activity. Food Chem. Toxicol. 2011, 49, 1096-1101. [CrossRef]

34. Fazio, A.; Plastina, P.; Meijerink, J.; Witkamp, R.F.; Gabriele, B. Comparative analyses of seeds of wild fruits of Rubus and Sambucus species from Southern Italy: Fatty acid composition of the oil, total phenolic content, antioxidant and anti-inflammatory properties of the methanolic extracts. Food Chem. 2013, 140, 817-824. [CrossRef]

35. Mladěnka, P.; Říha, M.; Martin, J.; Gorová, B.; Matějíček, A.; Spilková, J. Fruit extracts of 10 varieties of elderberry (Sambucus nigra L.) interact differently with iron and copper. Phytochem. Lett. 2016, 18, 232-238. [CrossRef]

36. Jakobek, L.; Šeruga, M.; Novak, I.; Medvidović-Kosanović, M. Flavonols, Phenolic Acids and Antioxidant Activity of Some Red Fruits. Dtsch. Lebensm. Rundsch. 2007, 103, 369-378.

37. Senica, M.; Stampar, F.; Veberic, R.; Mikulic-Petkovsek, M. Processed elderberry (Sambucus nigra L.) products: A beneficial or harmful food alternative? LWT Food Sci. Technol. 2016, 72, 182-188. [CrossRef]

38. Negahdari, R.; Bohlouli, S.; Sharifi, S.; Maleki Dizaj, S.; Rahbar Saadat, Y.; Khezri, K.; Jafari, S.; Ahmadian, E.; Jahandizi, N.G.; Raeesi, S. Therapeutic benefits of rutin and its nanoformulations. Phytother Res. 2020. [CrossRef]

39. Tarko, T.; Duda-Chodak, A. Influence of Food Matrix on the Bioaccessibility of Fruit Polyphenolic Compounds. J. Agric. Food Chem. 2020, 68, 1315-1325. [CrossRef]

40. Vrchotova, N.; Dadakova, E.; Matejicek, A.; Triska, J.; Kaplan, J. Effect of variety on content of bioactive phenolic compounds in common elder (Sambucus nigra L.). Nat. Prod. Res. 2017, 31, 700-703. [CrossRef]

41. Thomas, A.L.; Byers, P.L.; Gu, S.; Avery, J.J.D.; Kaps, M.; Datta, A.; Fernando, L.; Grossi, P.; Rottinghaus, G.E. Occurrence of Polyphenols, Organic Acids, and Sugars among Diverse Elderberry Genotypes Grown in Three Missouri (USA) Locations. Acta Hortic. 2015, 1061, 147-154. [CrossRef]

42. Veberic, R.; Jakopic, J.; Stampar, F.; Schmitzer, V. European elderberry (Sambucus nigra L.) rich in sugars, organic acids, anthocyanins and selected polyphenols. Food Chem. 2009, 114, 511-515. [CrossRef]

43. Petrut, G.; Muste, S.; Muresan, C.; Paucean, A.; Muresan, A.; Nagy, M. Chemical Profiles and Antioxidant Activity of Black Elder (Sambucus Nigra L.)—A Review. Bull. Univ. Agric. Sci. Vet. Med. Cluj Napoca Food Sci. Technol. 2017, 74. [CrossRef]

44. Kumar, S.; Pandey, A.K. Chemistry and biological activities of flavonoids: An overview. Sci. World J. 2013, 2013, 162750. [CrossRef]

45. Masek, A.; Latos-Brozio, M. The Effect of Substances of Plant Origin on the Thermal and Thermo-Oxidative Ageing of Aliphatic Polyesters (PLA, PHA). Polymer 2018, 10, 1252. [CrossRef]

46. Barbeş, L.; Bărbulescu, A.; Stanciu, G. Statistical analysis of mineral elements content in different melliferous plants from the dobrogea region, România. Rom. Rep. Phys. 2020, 72, 705.

47. Regulation (EU) No 1169/2011 of the European Parliament and of the Council of 25 October 2011 on the Provision of Food Information to Consumers, Annex XIII.: OJL 2011. Volume 304, p. 18. Available online: https: / / eur-lex.europa.eu/LexUriServ / LexUriServ.do?uri=OJ:L:2011:304:0018:0063:en:PDF (accessed on 25 May 2021). 